\title{
Multifractal Analysis of Pore Structure and Evaluation of Deep- Buried Cambrian Dolomite Reservoir with Image Processing: A Case from Tarim Basin, NW China
}

\author{
Xiaojun Zhang, ${ }^{1,2}$ Haodong Han $\mathbb{D}^{3}{ }^{3}$ Jun Peng $\mathbb{D}^{4},{ }^{4}$ and Yingchun Gou ${ }^{1,2}$ \\ ${ }^{1}$ Research Institute of Petroleum Exploration \& Development-Northwest, Petrochina, Lanzhou 730020, China \\ ${ }^{2}$ Key Laboratory of Reservoir Characterization, CNPC, Lanzhou 730020, China \\ ${ }^{3}$ Chengdu Center of China Geological Survey, Chengdu 610081, China \\ ${ }^{4}$ College of Geosciences and Technology, Southwest Petroleum University, Chengdu 610500, China
}

Correspondence should be addressed to Haodong Han; hanhaodong@163.com

Received 17 December 2019; Revised 6 July 2020; Accepted 10 July 2020; Published 21 September 2020

Academic Editor: Wei Wei

Copyright ( 92020 Xiaojun Zhang et al. This is an open access article distributed under the Creative Commons Attribution License, which permits unrestricted use, distribution, and reproduction in any medium, provided the original work is properly cited.

Reservoir pore space assessment is of great significance for petroleum exploration and production. However, it is difficult to describe the pore characteristics of deep-buried dolomite reservoirs with the traditional linear method because these rocks have undergone strong modification by tectonic activity and diagenesis and show significant pore space heterogeneity. In this study, 38 dolostone samples from 4 Cambrian formations of Tarim Basin in NW China were collected and 135 thin section images were analyzed. Multifractal theory was used for evaluation of pore space heterogeneity in deep-buried dolostone based on thin section image analysis. The physical parameters, pore structure parameters, and multifractal characteristic parameters were obtained from the digital images. Then, the relationships between lithology and these parameters were discussed. In addition, the pore structure was classified into four categories using $K$-means clustering analysis based on multifractal parameters. The results show that the multifractal phenomenon generally exists in the pore space of deep-buried dolomite and that multifractal analysis can be used to characterize the heterogeneity of pore space in deep-buried dolomite. For these samples, multifractal parameters, such as $\alpha_{\min }, \alpha_{\max }, \Delta \alpha_{\mathrm{L}}, \Delta \alpha_{\mathrm{R}}, \Delta f$, and AI, correlate strongly with porosity but only slightly with permeability. However, the parameter $\Delta \alpha$, which is usually used to reveal heterogeneity, does not show an obvious link with petrophysical properties. Of dolomites with different fabrics, fine crystalline dolomite and medium crystalline dolomite show the best petrophysical properties and show significant differences in multifractal parameters compared to other dolomites. More accurate porosity estimations were obtained with the multifractal generalized fractal dimension, which provides a new method for porosity prediction. The various categories derived from the $K$-means clustering analysis of multifractal parameters show distinct differences in petrophysical properties. This proves that reservoir evaluation and pore structure classification can be accurately performed with the $K$-means clustering analysis method based on multifractal parameters of pore space in deepburied dolomite reservoirs.

\section{Introduction}

Deep-buried dolomite reservoirs have strong heterogeneity and complex pore structure [1]. Their pore geometry and structure are strongly influenced by tectonic factors, diagenesis, and chemical reactions between rock and different fluids $[2,3]$. And they generally show various types of pore space, complex pore structure, and strong reservoir heterogeneity [1]. It will increase the difficulty of characterization on pore structure, feature description, and reservoir evaluation of deep-buried dolomite reservoirs.

It is well known that microscopic pore structure features control the reservoir quality. Therefore, clarifying the microscopic characteristics and heterogeneity of pore structure is very important for understanding the macroscopic characteristics of a reservoir [4]. However, it is difficult to characterize micropores' heterogeneity with traditional Euclidean geometry. Fortunately, fractal geometry, 


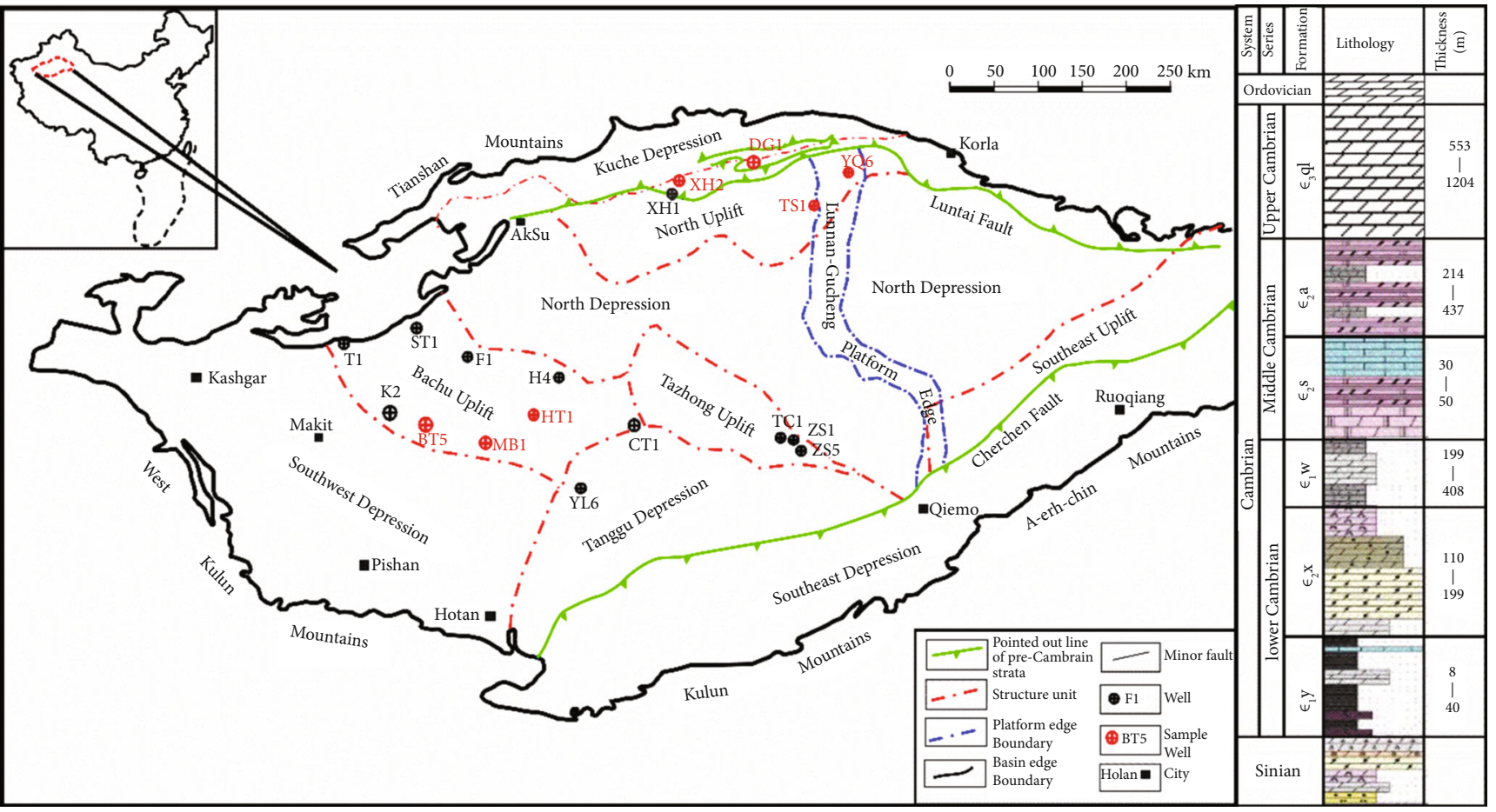

Figure 1: Basic information of the study area and distribution of the sampling well.

created by Mandelbrot [5], has been introduced into analyses of the pore distribution properties of sedimentary rocks, and it plays an important role in characterization of heterogeneity and self-similarity [6-8]. Compared to classical geometry, fractal geometry reveals the deterministic and stochastic unity laws of regularity and chaos in pore structure [9-14]. Furthermore, multifractal analysis is a multiscale method based on power-law relationships and can describe local irregular fluctuations more effectively than the monofractal method [15-17]. In multifractal analysis, the self-similarity measure can be regarded as the singularity strength and parameters of the multifractal spectrum through scale decomposition of interrelated fractal series [17-20]. To date, multifractal analysis has been widely used to depict the statistical properties of scale variation for studies in soil science, geosciences, and materials due to its advantage in heterogeneity analysis [21-27].

Fractal and multifractal behavior has been observed in sedimentary rocks and soil from scanning electron microscopy (SEM) [6, 28, 29], X-ray computed tomography (CT) $[12,30]$, nuclear magnetic resonance (NMR) $[18,31,32]$, and mercury intrusion analysis of core plugs [23, 33-36]. Fractal geometry and the multifractal method have been widely used to describe the porosity and permeability of porous media and clastic rocks [37-40]. However, only a few of these studies have focused on carbonate rocks [16, $20,41-44]$, and research on deep-buried dolomite is even more limited. Accordingly, further study on deep-buried dolomites within the framework of multifractal theory is needed in an attempt to expand and deepen the application of this powerful mathematics tool in different fields of the earth sciences. Meanwhile, a new evaluation method based on multifractal theory is proposed to study the characteristics of pore space and pore structure in dolomite reservoirs.

Thin section analysis is an easy way to determine the two-dimensional topological properties of pore space and pore throat size in rocks, despite its lower resolution and magnitude compared to SEM and CT. In this study, multifractal analysis and digital image analysis of thin section images were used to extract quantitative information reflecting the distribution of pore space and the pore structure characteristics. Thirty-eight Cambrian dolomite core plug samples from Tarim Basin in northwestern China were chosen as the study objects, and casting thin section images were obtained. Then, based on image analysis, the multifractal behavior of pore space in deep-buried dolomite and the application of multifractal theory for heterogeneity evaluation were analyzed, and the relationships between multifractal parameters and the physical properties of different lithologies were studied. Finally, classification of the pore structure was accomplished with $K$-means clustering analysis of multifractal parameters. The results are of great significance for quantitative heterogeneity characterization, pore structure evaluation, and petroleum exploration in deep-buried dolomite reservoirs.

\section{Methodology}

2.1. Study Area and Sampling. Tarim Basin located in northwestern China is the largest inland basin with total area of $53 \times 10^{4} \mathrm{~km}^{2}$ in China. It is bounded by West Kunlun Mountains, Tianshan Mountains, Kunlun Mountains, and Altun Mountains (Figure 1). For detailed geological setting and stratigraphic column of the basin, the reader can refer to Du and 
Pan [45]. In the study, up to 38 dolostone samples were collected from different formations of the Cambrian strata in 7 wells to estimate the petrophysical properties. These samples were made into a plunger for the petrophysical test and molded into thin section for image processing, respectively. The information of each sample could be seen in Table 1.

2.2. Experiments. Helium porosity and permeability were measured by CMS-300 Automated Permeameter (Core Measurement Systems) under confining pressure of 34.5 $\mathrm{MPa}$. For image analysis, casting thin sections were produced with the following procedure: (1) set samples in a glass tube at constant temperature of $100^{\circ} \mathrm{C}$ for $2 \mathrm{~h}$; (2) reduce the glass tube to a vacuum with pressure $0.09 \mathrm{MPa}$ and keep samples in it for $1 \mathrm{~h}$; (3) inject blue epoxy resin into samples at pressure of $8 \mathrm{MPa}$ and temperature of $100^{\circ} \mathrm{C}$ for $12 \mathrm{~h}$; and (4) after natural cooling, polish the samples into thin sections with thickness $20 \mu \mathrm{m}$, and mount each chip on a glass slide. Therefore, pore spaces in thin section were filled by blue epoxy resin and could be easily distinguished from matrix under the microscope. For image and multifractal analyses, chromophotographs were captured from representative views of each thin section. These collected images with different magnifications, such as $25 \mathrm{x}, 50 \mathrm{x}, 100 \mathrm{x}$, and $400 \mathrm{x}$, can reveal both macropores and micropores. All images were obtained using the transmission mode of BX51 microscope in State Key Laboratory of Oil and Gas Reservoir Geology and Exploitation, Southwest Petroleum University, China.

2.3. Image Analysis. Casting thin section observation is an inexpensive tool for characterizing pore structure in rocks and has been widely used in heterogeneity of rock microscopic pores [44]. By image processing on thin section image, the pore type, pore shape, pore scale, and pore throat type could be acquired. The image analysis consists of image filtering, image segmentation, connected domain identification, and statistics of pore size distribution (PSD). First, median filtering was used to eliminate noise in images. The $K$-nearest neighbor (KNN) classification algorithm is implemented in image segmentation for getting binary image, where the pixel values one and zero represent pore and matrix, respectively. Then, the coordinates and area of each pore were obtained with the connected domain identification method [46]. Finally, parameters such as total porosity $(\varphi)$, PSD, average pore radius $\left(r_{\mathrm{b}}\right)$, average pore throat radius $\left(r_{t}\right)$, and permeability $(K)$ value were calculated with the following formulas [47-50].

$$
\begin{gathered}
\varphi=\frac{A_{\text {pore }}}{A_{\text {total }}}, \\
r_{\mathrm{b}}=\frac{\sum_{i=1}^{\mathrm{Num}} r_{i} A_{i}}{A_{\text {total }}}, \\
r_{\mathrm{t}}=\frac{\sum_{i=1}^{\mathrm{Num}} r_{\mathrm{t} i} A_{i}}{A_{\text {total }}},
\end{gathered}
$$

where $A_{\text {pore }}$ is the pore area in the image, $A_{\text {total }}$ is the total area of the image, $r_{i}$ is the mean radius of each pore, $A_{i}$ is the area of $i$ th pore, Num is the total number of pores, and $r_{t \mathrm{i}}$ is the minimum radius of each pore.

According to the values of $\varphi, r_{\mathrm{b}}$, and $r_{\mathrm{t}}$, the permeability value $K$ could be obtained with the following formula [51]:

$$
K=\frac{\varphi r_{\mathrm{b}}^{2}}{\tau^{2}} \cdot \frac{\left[\ln \left(r_{\mathrm{b}} / r_{\mathrm{t}}\right)\right]^{2}}{\left[\left(r_{\mathrm{b}} / r_{\mathrm{t}}\right)^{2}-1\right] \cdot\left[\left(r_{\mathrm{b}} / r_{\mathrm{t}}\right)^{2}-\left(r_{\mathrm{t}} / r_{\mathrm{b}}\right)^{2}\right]},
$$

where $\tau$ is pore tortuosity in samples, and here, its value was assumed to be 1 . The estimated values of porosity and permeability are shown in Table 1.

2.4. Multifractal Analysis. For multifractal analysis, a cubic box of size $\delta$ was used to cover the pore space. Assuming that there are $m_{i}$ pore pixels in the $i^{\text {th }}$ box $(1 \leq i \leq n(\delta)), n(\delta)$ denotes the number of boxes required to cover the pore space at the scale of $\delta$ and the total number of pixels in the pore space is $M$. The measurement $\mu_{i}(\delta)$, which denotes the local probability of finding a box to cover the pore space at the scale of $\delta$, can be defined as follows [52]:

$$
\mu_{i}=\frac{m_{i}}{M}
$$

With respect to the fluctuation in local porosity, a local crowding index $\alpha_{i}$ can be defined for the $i^{\text {th }}$ box, i.e., the singularity exponent, and it holds that

$$
\mu_{i}(\delta) \propto \delta^{\alpha_{i}}
$$

Two approaches are available to compute the multifractal spectrum: the moment method and the direct method [52]. In both methods, a weighted sum over all boxes must be performed to yield the partition function $\chi(q, \delta)$ as follows:

$$
\chi(q, \delta)=\sum_{i}\left[\mu_{i}(\delta)\right]^{q}=\sum_{i}\left(\frac{m_{i}}{M}\right)^{q}
$$

where the variable $q$ denotes the order of moment for $\mu_{i}$; it is clear to note that different $q$ values actually allow probing of the contribution to $\chi(q, \delta)$ from boxes with different $\mu_{i}$. In particular, at negative $q$ values, $\chi(q, \delta)$ is dominated by boxes with small $\mu_{i}$, while at positive $q$ values, $\chi(q, \delta)$ is dominated by boxes with large $\mu_{i}$. For a multifractal measure, it holds that

$$
\chi(q, \delta) \propto \delta^{\tau(q)}
$$

where the mass exponent $\tau(q)$ is a nonlinear function of $q$ but independent on $\delta$; the singularity exponent $\alpha$ and the Hausdorff dimension $f(a)$ can be determined with the Legendre transformation:

$$
\alpha(q)=\frac{d \tau(q)}{d q}
$$


TABLE 1: Basic information, petrophysical data from helium porosimetry, and image analysis of samples in the study.

\begin{tabular}{|c|c|c|c|c|c|c|c|c|c|c|}
\hline Sample & Well & Depth & Formation & Lithology & Image Number & $\varphi(\%)$ & $\varphi_{\mathrm{I}}(\%)$ & $\varphi_{M}(\%)$ & $K(\mathrm{mD})$ & $K_{\mathrm{I}}(\mathrm{mD})$ \\
\hline S1 & BT5 & 4812.9 & $\epsilon_{3} \mathrm{ql}$ & $\mathrm{MD}$ & 4 & 0.80 & 2.99 & 0.79 & 0.001 & 0.809 \\
\hline S2 & BT5 & 5782.08 & $\epsilon_{1} \mathrm{x}$ & $\mathrm{MD}$ & 6 & & 7.48 & 2.01 & & 0.889 \\
\hline S3 & BT5 & 5783.95 & $\epsilon_{1} \mathrm{x}$ & MD & 4 & 4.30 & 8.56 & 2.83 & 0.540 & 3.072 \\
\hline S4 & BT5 & 5784.8 & $\epsilon_{1} \mathrm{x}$ & $\mathrm{MD}$ & 6 & 2.50 & 10.20 & 2.74 & 0.01 & 0.558 \\
\hline S5 & BT5 & 5785.12 & $\epsilon_{1} \mathrm{x}$ & $\mathrm{FD}$ & 4 & 3.30 & 14.87 & 4.29 & 1.160 & 7.365 \\
\hline S6 & BT5 & 5785.95 & $\epsilon_{1} \mathrm{x}$ & FD & 6 & & 7.24 & 3.07 & & 1.703 \\
\hline S7 & DG1 & 6216.87 & $\epsilon_{3} \mathrm{ql}$ & SD & 3 & 1.00 & 2.06 & 2.27 & 0.020 & 0.011 \\
\hline S8 & DG1 & 6217.99 & $\epsilon_{3} \mathrm{ql}$ & GD & 2 & 1.00 & 0.96 & 0.97 & 0.020 & 0.059 \\
\hline S9 & DG1 & 6256.34 & $\epsilon_{3} \mathrm{ql}$ & FD & 4 & 2.70 & 12.25 & 3.17 & 22.600 & 3.587 \\
\hline S10 & DG1 & 6295.28 & $\epsilon_{3} \mathrm{ql}$ & FD & 3 & & 5.46 & 1.73 & & 0.644 \\
\hline S11 & DG1 & 6297.6 & $\epsilon_{3} \mathrm{ql}$ & FD & 2 & & 1.83 & 0.81 & & 0.531 \\
\hline S12 & HT1 & 6163.85 & $\epsilon_{2} \mathrm{a}$ & GD & 2 & 0.90 & 0.86 & 0.48 & 20.877 & 0.013 \\
\hline S13 & $\mathrm{XH} 2$ & 5350.45 & $\epsilon_{3} \mathrm{ql}$ & FD & 5 & 4.80 & 18.40 & 5.24 & 4.050 & 2.977 \\
\hline S14 & $\mathrm{XH} 2$ & 5355.3 & $\epsilon_{3} \mathrm{ql}$ & SD & 4 & & 13.98 & 5.78 & & 1.627 \\
\hline S15 & $\mathrm{XH} 2$ & 5515.87 & $\epsilon_{3} \mathrm{ql}$ & SD & 3 & & 3.36 & 0.79 & & 0.439 \\
\hline S16 & $\mathrm{XH} 2$ & 5517.68 & $\epsilon_{3} \mathrm{ql}$ & $\mathrm{FD}$ & 5 & & 4.32 & 2.17 & & 0.153 \\
\hline S17 & $\mathrm{XH} 2$ & 5590.4 & $\epsilon_{3} \mathrm{ql}$ & DM & 2 & & 0.83 & 1.13 & & 0.002 \\
\hline S18 & $\mathrm{XH} 2$ & 5590.9 & $\epsilon_{3} \mathrm{ql}$ & DM & 2 & & 1.38 & 1.74 & & 0.005 \\
\hline S19 & $\mathrm{XH} 2$ & 5595.76 & $\epsilon_{3} \mathrm{ql}$ & GD & 5 & & 4.43 & 1.76 & & 0.527 \\
\hline S20 & YQ6 & 7059.35 & $\epsilon_{3} \mathrm{ql}$ & $\mathrm{CD}$ & 3 & & 3.50 & 2.42 & & 0.240 \\
\hline S21 & YQ6 & 7060.43 & $\epsilon_{3} \mathrm{ql}$ & $\mathrm{CD}$ & 3 & & 2.43 & 1.48 & & 0.364 \\
\hline S22 & YQ6 & 7061.07 & $\epsilon_{3} \mathrm{ql}$ & $\mathrm{CD}$ & 5 & 1.80 & 2.21 & 1.77 & 1.350 & 0.099 \\
\hline S23 & YQ6 & 7062.3 & $\epsilon_{3} \mathrm{ql}$ & $\mathrm{MD}$ & 3 & 0.30 & 1.43 & 0.51 & 0.060 & 0.132 \\
\hline S24 & YQ6 & 7118.5 & $\epsilon_{3} \mathrm{ql}$ & $\mathrm{MD}$ & 5 & 2.20 & 11.45 & 3.52 & 0.450 & 1.187 \\
\hline S25 & YQ6 & 7118.78 & $\epsilon_{3} \mathrm{ql}$ & FD & 3 & 2.90 & 3.58 & 1.28 & 0.130 & 0.590 \\
\hline S26 & YQ6 & 7119.3 & $\epsilon_{3} \mathrm{ql}$ & $\mathrm{MD}$ & 3 & 2.70 & 2.69 & 1.46 & 0.020 & 0.327 \\
\hline S27 & YQ6 & 7119.42 & $\epsilon_{3} \mathrm{ql}$ & FD & 4 & 0.80 & 1.32 & 1.08 & 0.700 & 0.006 \\
\hline S28 & YQ6 & 7119.93 & $\epsilon_{3} \mathrm{ql}$ & FD & 4 & 2.80 & 5.03 & 2.82 & 0.070 & 1.552 \\
\hline S29 & YQ6 & 7314.05 & $\epsilon_{3} \mathrm{ql}$ & SD & 3 & 0.60 & 1.11 & 0.55 & 0.003 & 0.033 \\
\hline S30 & YQ6 & 7313.74 & $\epsilon_{3} \mathrm{ql}$ & SD & 3 & 0.60 & 2.13 & 0.42 & 0.003 & 0.331 \\
\hline S31 & BT5 & 4811.6 & $\epsilon_{3} \mathrm{ql}$ & FD & 3 & 0.20 & 1.57 & 1.08 & & 0.034 \\
\hline S32 & MB1 & 6002.98 & $\epsilon_{2} \mathrm{~s}$ & $\mathrm{SD}$ & 3 & 2.40 & 5.07 & 3.58 & 12.800 & 0.089 \\
\hline S33 & MB1 & 6008.59 & $\epsilon_{2} \mathrm{~s}$ & $\mathrm{SD}$ & 3 & 5.80 & 5.55 & 6.42 & 3.170 & 0.097 \\
\hline S34 & MB1 & 6009.07 & $\epsilon_{2} \mathrm{~s}$ & GD & 3 & 3.20 & 1.70 & 1.44 & 0.740 & 0.024 \\
\hline S35 & TS1 & 7105.43 & $\epsilon_{3} \mathrm{ql}$ & DM & 3 & 0.6 & 1.04 & 1.16 & 0.6 & 0.913 \\
\hline S36 & TS1 & 7268.10 & $\epsilon_{3} \mathrm{ql}$ & FD & 2 & 3.7 & 2.92 & 1.73 & 0.03 & 2.677 \\
\hline S37 & TS1 & 7875.60 & $\epsilon_{3} \mathrm{ql}$ & FD & 4 & 3.7 & 8.09 & 4.39 & 34.14 & 3.772 \\
\hline S38 & TS1 & 8407.56 & $\epsilon_{3} \mathrm{ql}$ & SD & 3 & 9.10 & 15.32 & 8.90 & 4.160 & 2.270 \\
\hline
\end{tabular}

The abbreviation " $\epsilon_{3} \mathrm{ql}$ " represents Lower Qiulitage Formation of Upper Cambrian; " $\epsilon_{2} s$ " represents Shayilik Formation of Middle Cambrian; and " $\epsilon_{1}$ " " represents Sholbrak Formation of Lower Cambrian. $\varphi_{\mathrm{I}}$ is the porosity obtained with Equation (1), $\varphi_{\mathrm{M}}$ is the porosity obtained with Equation (16), and $K_{\mathrm{I}}$ is the permeability computed from Equations (2)-(4) with images.

$$
f(\alpha(q))=q \cdot \alpha(q)-\tau(q)
$$

The multifractal generalized dimensions $D_{q}$ of the $q^{\text {th }}$ order are defined as

$$
D_{q}=\frac{1}{q-1} \lim _{\delta \rightarrow 0} \frac{\ln [\chi(q, \delta)]}{\ln (\delta)}
$$

When $q=1$, the above equation can be expressed as

$$
D_{1}=\lim _{\delta \rightarrow 0}\left(\frac{\sum_{i=1}^{N(\delta)} \mu_{i}(\delta) \ln \left(\mu_{i}(\delta)\right)}{\ln (\delta)}\right)
$$

The multifractal generalized dimensions $D_{q}$ are related to the mass exponent $\tau(q)$ by 


$$
\tau(q)=(q-1) D_{q} .
$$

The $q-D_{q}, q-\alpha$, and $\alpha-f(\alpha)$ construct the powerful tool for characterizing the heterogeneity of pore structure.

2.5. Porosity Estimation Based on Multifractal Analysis. It is clear that the porosity of dolomite can be estimated using the total number and area of boxes as follows:

$$
\phi=\frac{N(\delta) \bullet \delta^{2}}{A_{\text {total }}},
$$

where $N(\delta)$ is the box number for pores, $\delta$ is the size of the box, and $A_{\text {total }}$ is the total area of the image. Using the boxcounting method for fractal and multifractal theory, the total number of boxes covering the pore space can be expressed as the following formula:

$$
N(\delta) \propto \delta^{-D_{0}},
$$

where $D_{0}$ is the capacity dimension obtained from Equation (11) when $q$ is equal to zero. Then, the porosity can be calculated with the box size $\delta$ and the capacity dimension $D_{0}$ as follows:

$$
\phi \propto \frac{\delta^{2-D}}{A_{\text {total }}} .
$$

In order to facilitate the calculation, $A_{\text {total }}$ was assumed to be 1 , and $\delta$ was set as the reciprocal of the pixel number for each column of the image. Therefore, the porosity can be estimated with the pixel number in the image and the capacity dimension.

\section{Results}

3.1. Porosity, Permeability, and Micropore Characteristics of Dolomite. Dolomite is widespread in Cambrian strata of Tarim Basin. According to classification and nomenclature schemes of carbonate rocks suggested by Dunham [53], the dolomite in the study area can be divided into dolomicrite (DM), crystalline dolomite, and grain dolomite (GD). In addition, crystalline dolomites are subdivided into silty crystalline dolomite (SD), fine crystalline dolomite (FD), medium crystalline dolomite (MD), and coarse crystalline dolomite (CD) in accordance with the crystal size.

The porosity determined from the helium porosimetry test shows that these samples have low porosity values ranging from $0.2 \%$ to $9.1 \%$ (Table 1 ) and wide permeability values ranging from $0.003 \mathrm{mD}$ to $34.14 \mathrm{mD}$. The Cambrian dolomite contains a variety of reservoir spaces such as vugs, intergranular pores, intergranular dissolved pores, and fractures. Intergranular or intercrystal dissolved pores are mainly developed in the silty, fine, and medium crystalline dolomites; intercrystalline pores mainly existed in the silty and fine crystalline dolomites; and fractures are widely distributed in various types of dolomites. Typically, intergranular dissolved pores are connected by microfractures or flat pore throats. In addition, some throats and pores filled by bitumen indicate that the rocks have experienced oil and gas accumulation and filling.

The shape and size of intergranular or intercrystal dissolved pores are mainly controlled by the crystal structure and morphology. In general, compared to the other dolomites, the fine crystalline dolomite and medium crystalline dolomite with high reservoir quality and permeability are the most favorable reservoir rocks. As shown in Figure 2, there are obvious differences in pore type and porosity between the various dolomite types constituting the Cambrian dolomite formations of Tarim Basin.

The microcrystalline dolomite is mainly formed in tidal flat environment with arid climate at the penecontemporaneous stage. It formed from the $\mathrm{Mg}^{2+}$ rich in brine replacing $\mathrm{Ca}^{2+}$ in marl sediment. Due to the relatively fast crystallization speed and without being affected by later diagenesis, microcrystalline dolomites are usually with fine, poor euhedral crystals and close contact. Its porosity and permeability are poor and with average value of $2.1 \%$ and $0.175 \mathrm{mD}$, respectively (Table 1). The microcrystalline dolomite often shows the structure of laminae and bird eyes and develops a small amount of cracks and sutures mostly filled by calcite, quartz, gypsum, and anhydrite (Figure 2(a)). Thus, it is difficult to become an effective reservoir.

Silty dolomite formed from the recrystallization of microcrystalline dolomite in shallow burial environment. It performs as small crystal size, poor subhedral crystal, dirty crystal surface, and with contact each other. Cloudy centers and clear borders can be seen in some silty dolomite crystals. In this type of dolomite, dissolved pores and intercrystalline and look like pinhole and with dense distribution. The pore space performs as irregular harbor and with homogeneous pore size (Figure 2(b)).

3.2. Pore Characteristics. Sedimentation is the most important factor, which provides the material foundation for the primary porosity and pore development in dolomite. Typically, carbonates with high primary porosity form in highenergy environments, such as granular beaches and microbial mounds. However, tectonic activity also plays an important role in modifying porosity and pore space in dolomite. For example, as a result of tectonic uplift, buried carbonate rocks may be exposed at the surface and dissolved by atmospheric freshwater or karstification, such that large amounts of intergranular dissolved pores form in reservoir rocks. Alternatively, in the vicinity of tectonic fault zones, intensive formation of concentrated fracture networks may provide channels for geofluid flow. Acids contained in these fluids can dissolve carbonate minerals, form pores along fractures, and increase the porosity. Finally, the comprehensive transformation and alteration by various diagenetic processes in dolomite determine the final state of the pore space and porosity in reservoir rocks.

The image analysis results show that significant differences in porosity and pore size distribution exist in deepburied dolomites with different crystal sizes and the pore size distribution reveals strong heterogeneity. As shown in Figure 3, the samples numbered S2, S32, and S22 are fine 


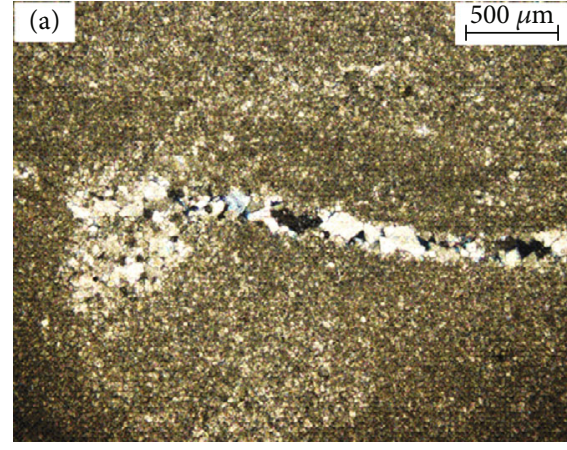

(a)

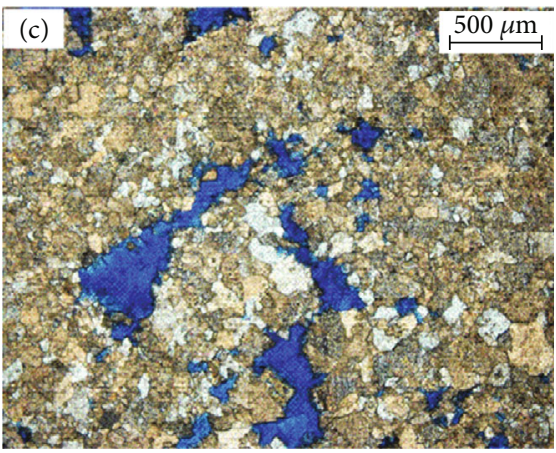

(c)

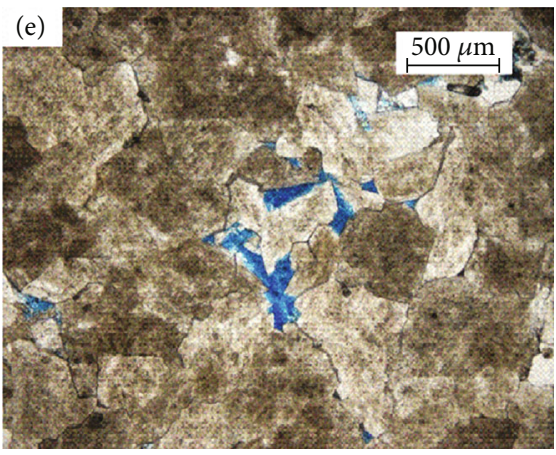

(e)

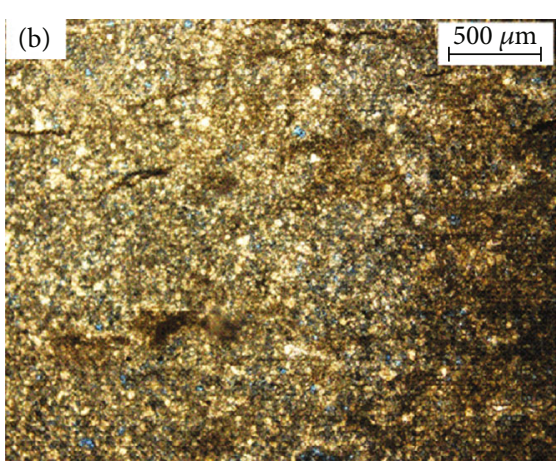

(b)

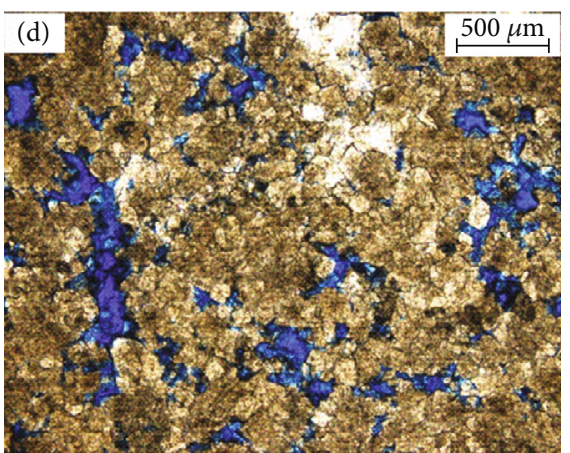

(d)

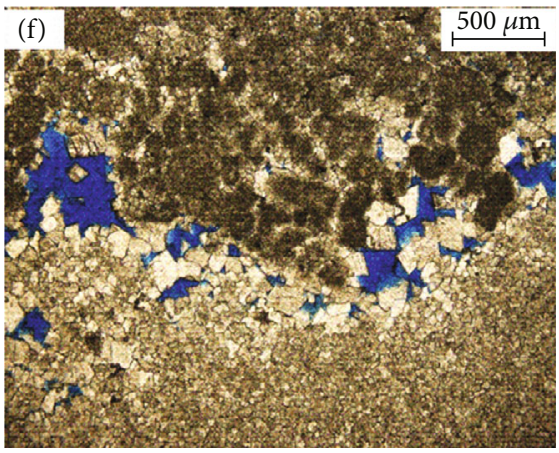

(f)

FIgURE 2: The thin section images of different types of dolomite. (a) Thin section image of MD sample S18 with low porosity value $1.38 \%$. It shows that the intergranular pores are not developed and the fractures are filled with quartz, asphalt, and other minerals. (b) Image of SD sample S32 with porosity 5.07\%. The pore space consists of medium and small intergranular pores and dissolved pores. (c) Image of FD sample S5 with porosity $14.87 \%$. The pores are mainly larger intergranular dissolved pores. (d) Image for MD sample S4 with porosity $10.2 \%$. Intergranular pores and intergranular dissolved pores with large size mainly develop in such type of dolomite. (e) Image of CD sample S22 with porosity $2.21 \%$. The intergranular pores were slightly developed. (f) Image of GD sample S19 with porosity $4.43 \%$. Dolarenite was recrystallized as fine crystalline dolomite. The intergranular dissolved pore with medium scale distributes along fractures.

crystalline dolomite, silty crystalline dolomite, and medium crystalline dolomite, respectively.

In the fine crystalline dolomite sample (S2), with porosity of $9.5 \%$, large intercrystalline pores are well developed. In the silty crystalline dolomite sample (S32), with porosity of $5.1 \%$, intercrystalline pores are well developed due to dissolution and dolomitization in the seepage-reflux model. As indicated by sample S32, the silty crystalline dolomite evolved from lagoon sediments containing gypsum deposited during evaporation. During the syngenetic period, the silty dolomite formed due to evaporation pump dolomitization. When sea level fell, the silty crystalline dolomite formation was exposed at the surface and underwent dissolution by freshwater, which formed the intergranular dissolved pores and gypsum mold pores. During the later burial stage, fractures formed due to tectonic activity and were filled by organic acid and asphalt from pyrolysis of hydrocarbons. The organic acid dissolved residual gypsum and soluble calcite, which increased the intergranular dissolved pores. In other words, the early gypsum and small dolomite crystal size determine the pore size ranging from small to medium scale. However, in the medium crystalline dolomite sample (S22) with a low porosity value of $2.3 \%$, only a few intergranular pores and microfractures were developed. Due to hydrothermal filling, 


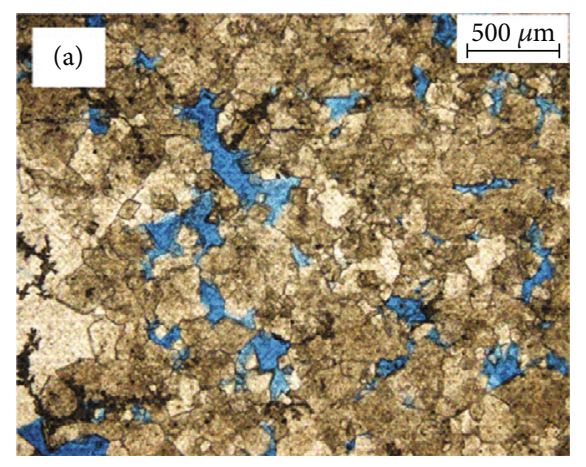

(a)

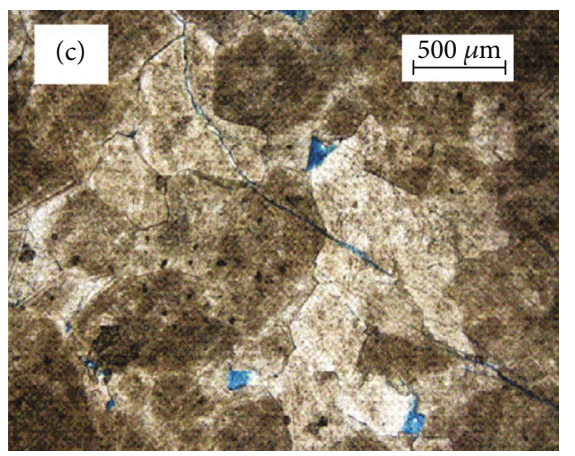

(c)

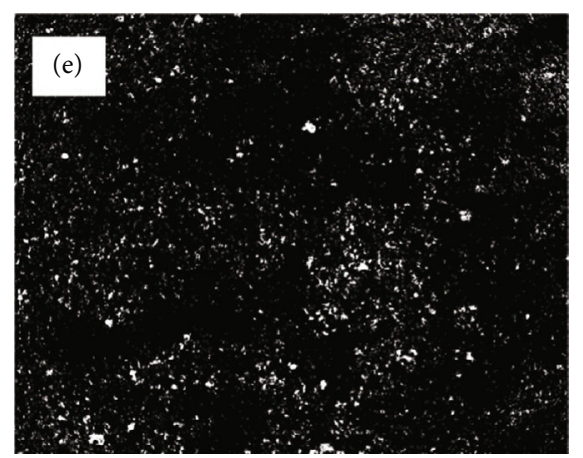

(e)

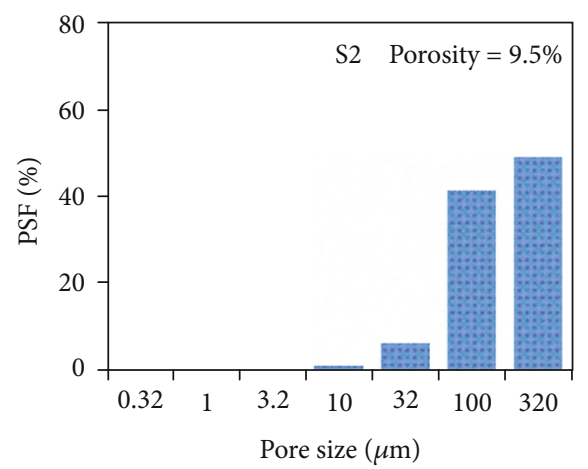

(g)

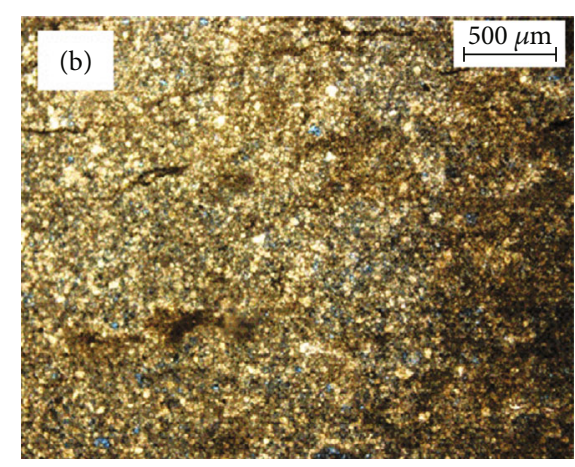

(b)

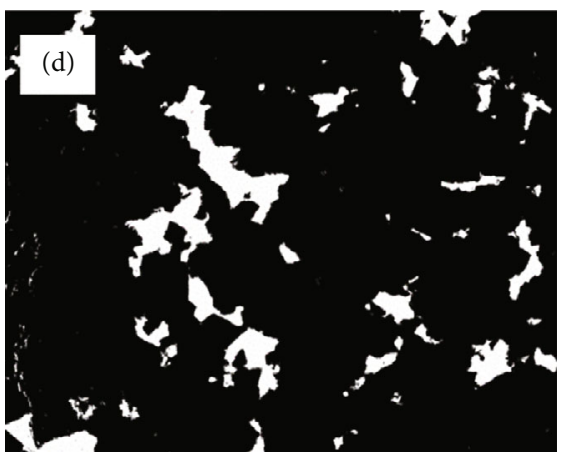

(d)

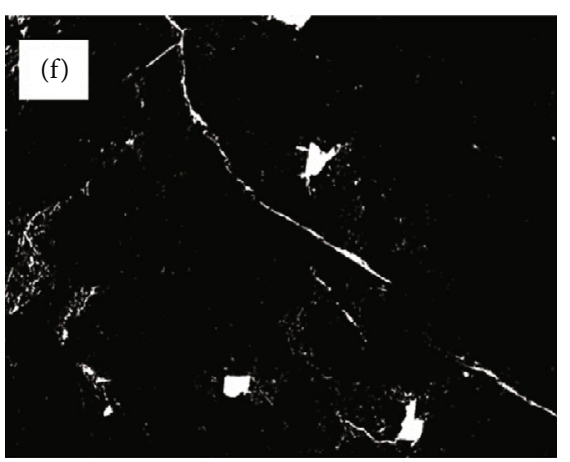

(f)

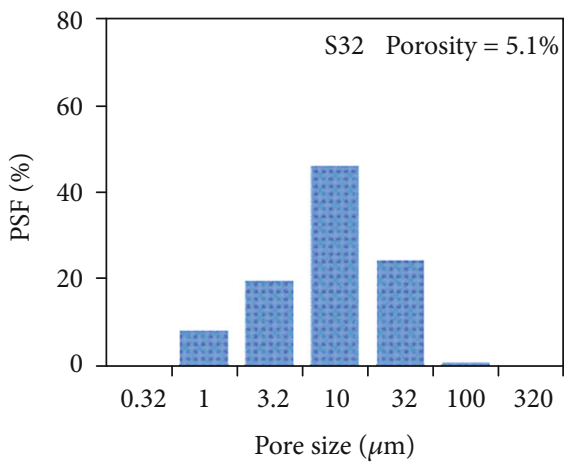

(h)

Figure 3: Continued. 


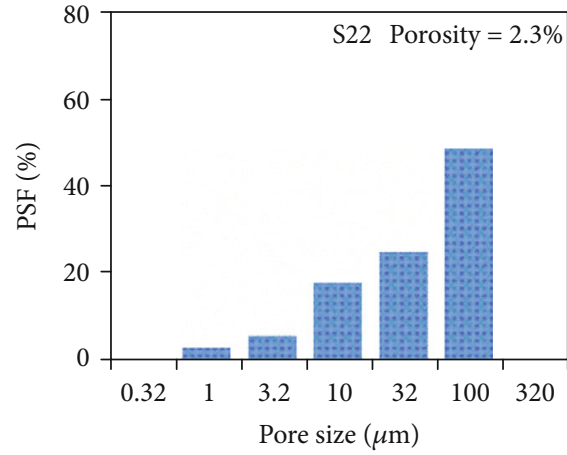

(i)

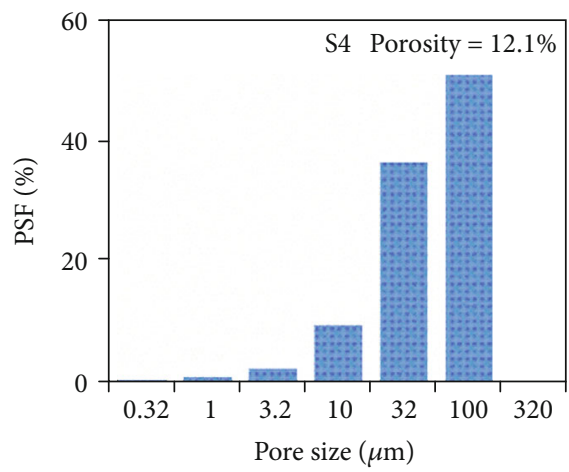

(k)

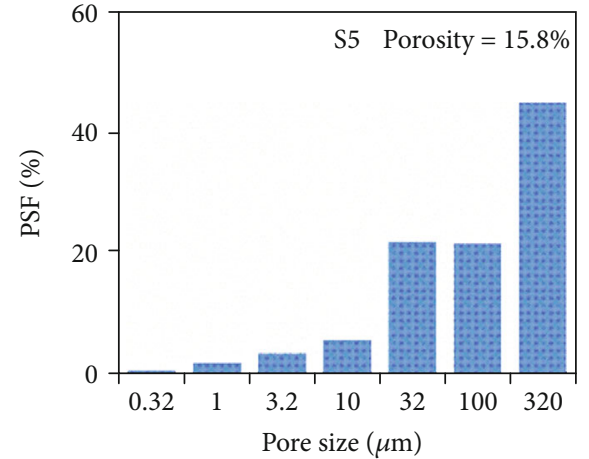

(j)

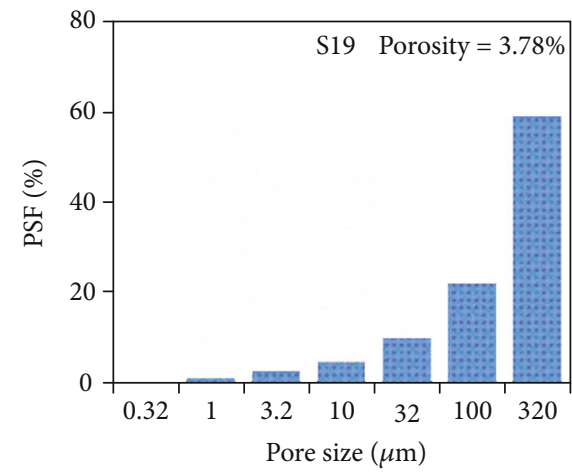

(1)

FIgURE 3: Thin section images, binary images, and pore size distribution of dolostone samples. (a) Intercrystalline pores and intercrystal dissolved pores in sample S2, well BT5, $5782.08 \mathrm{~m}, \epsilon_{1} \mathrm{x}$, medium crystalline dolomite. (b) Intercrystal dissolved pores in sample S32, well MB1, $6002.98 \mathrm{~m}, \epsilon_{2} \mathrm{~s}$, silty crystalline dolomite. (c) Fracture and intercrystal dissolved pores in sample S22, well YQ6, $7061.07 \mathrm{~m}, \epsilon_{3} \mathrm{ql}$, coarse crystalline dolomite. (d-f) The binary image of samples S2, S32, and S22, where the white and black pixel represents pore and solid matrix, respectively. (g-l) The pore size distribution and porosity of samples S2, S32, S22, S5, S4, and S19 corresponding to images in Figure 2 and this figure.

pores in the coarse crystalline dolomite were filled by large saddle dolomite crystals and were severely diminished. Only a few fine pores and fractures remained. These fractures and dissolved pores were formed under late stress conditions and were distributed along the grain boundaries.

The pore size distributions in samples S22 and S32 show a wide scale range and stronger heterogeneity than that in sample S2. The tiny crystals in sample S32 determine the pore scale, producing small- and medium-scale pores, whereas in the coarse crystalline dolomite, the coarse crystals are more difficult to dissolve completely but easily form fractures and dissolved pores along the crystal edges under stress action and dissolution (Figure 3 ).

3.3. Multifractal Analysis on Pores of Dolomite. In this study, the order of moment $q$ is selected from -5 to 5 with steps of 0.5 . The value of $\chi(q, \delta)$ at $q$ of $\pm 5, \pm 4, \pm 3, \pm 2, \pm 1$, and 0 are shown in Figure $4(\mathrm{a})$. The values $\tau(q)$, the slope of these $\chi(q, \delta)-q$ lines, can be determined with the least square fitting method, as shown in Figure 4(b). Therefore, the singularity exponent $\alpha(q)$ and multifractal spectrum $f(\alpha)$ can be obtained with the direct method via Equations (9) and (10), and results are shown in Figures 4(c) and 4(d). The program for calculating the fractal dimension, singularity exponent, mass exponent, and multifractal spectrum of pores in dolomite from thin section images has been designed. The characteristic parameters of multifractal spectra were extracted from binary images with a batch process using this software, and the spectrum plots $\alpha(q)-q, D(q)-q, \tau(q)-q$, and $f(\alpha)-\alpha$ for each image were exported in batch.

The most popular parameters for pore structure characterization are $D_{q}, f(\alpha), \tau(q)$, and $\Delta \alpha$. In general, $D_{q}$ increases with increasing pore structure complexity [19]. $\Delta \alpha$ is often used as an indicator of the heterogeneity and is positively correlated with heterogeneity. Corresponding to $q=0,1$, and 2 , the generalized dimension $D_{q}$ is the capacity dimension, information dimension, and correlation dimension, respectively.

In multifractal analysis, the singularity exponent $\alpha$ and the multifractal spectrum can be used to describe local material features. The continuous distribution of $\alpha$ may be used to depict the pore structure of rocks, with the parameters $\alpha_{\text {min }}$ and $\alpha_{\max }$ corresponding to the maximum and minimum pore sizes, respectively, as shown in Figure 4(c). The width of the multifractal spectrum $\left(\Delta \alpha=\alpha_{\max }-\alpha_{\text {min }}\right)$ reflects the irregularity strength of the probability measure for a physical quantity in the whole fractal structure and reveals fractal 


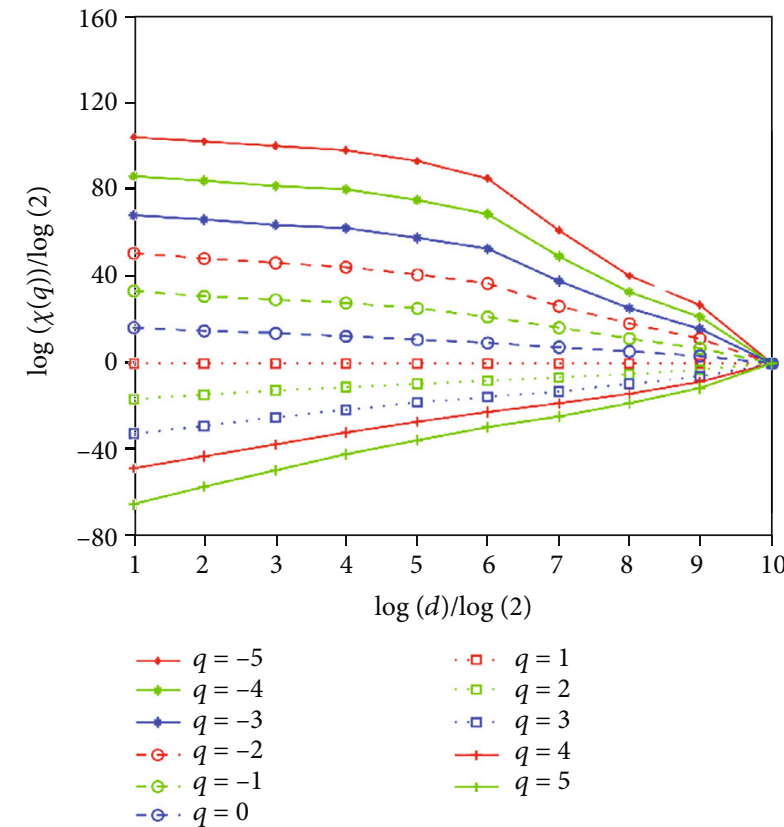

(a)

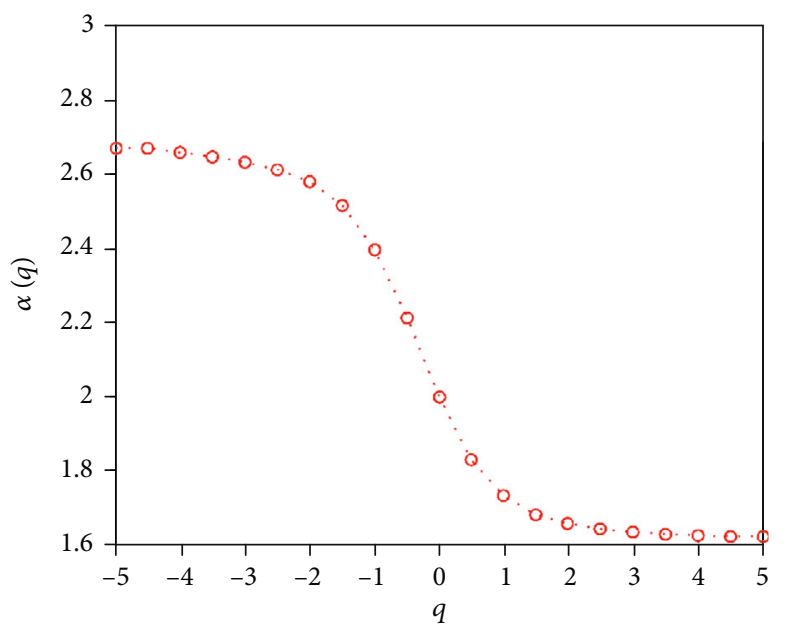

(c)

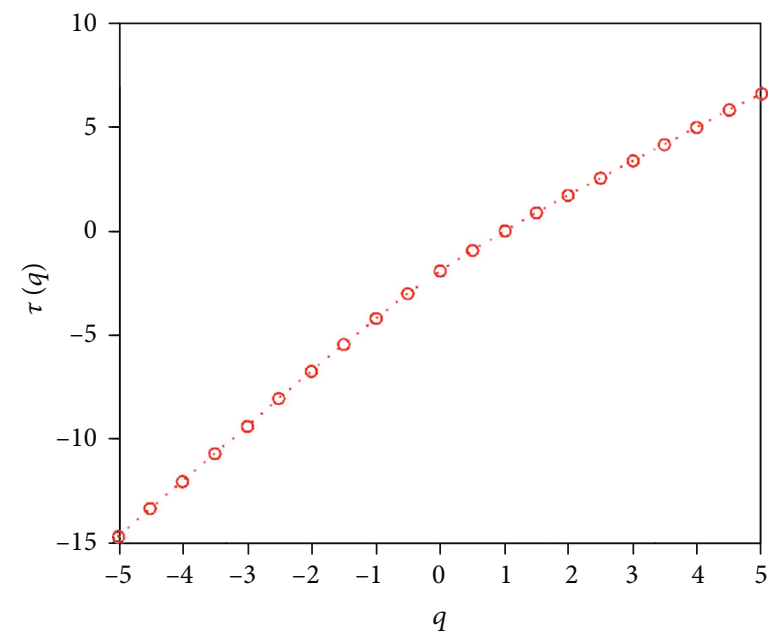

(b)

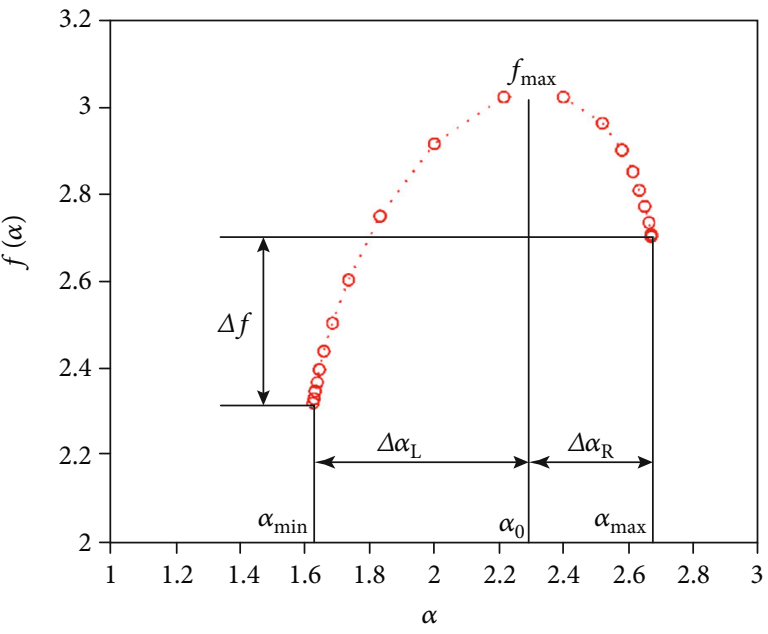

(d)

Figure 4: Procedures of multifractal analysis on pore space in dolomites. (a) The partition function of probability to cover the pore space at different $q$ values. (b) The mass exponent $\tau$ varying with the moment $q$. (c) The singularity exponent $\alpha$ computed from the direct method. (d) The multifractal spectrum.

characteristics at different scales and local conditions. The wider the $f(\alpha)-\alpha$ spectrum, the higher the heterogeneity in the local scaling indices of the studied variable and vice versa. Thus, a higher value of $\Delta \alpha$ indicates a more heterogeneous distribution of the probability measure for a physical quantity. The parameter $\Delta f$ is the amplitude difference between the values of function $f(\alpha)$ at $\alpha_{\max }$ and $\alpha_{\min }$ and indicates the morphological characteristics of the multifractal spectrum and the proportion between the maximum and minimum singularity, as shown in Figure $4(\mathrm{~d})$. The value $\Delta \alpha_{\mathrm{L}}$ is the difference between $\alpha_{\min }$ and $\alpha_{0}$, which is the width of the left half of the multifractal spectrum from $\alpha_{\min }$ to $\alpha_{0}$, and the value $\Delta \alpha_{\mathrm{R}}$ is the right half width. Similarities and differences of pore distribution in rocks can be also be examined with the help of the asymmetrical index (AI), which is the ratio between $\left(\Delta \alpha_{\mathrm{L}}-\Delta \alpha_{\mathrm{R}}\right)$ and $\left(\Delta \alpha_{\mathrm{L}}+\Delta \alpha_{\mathrm{R}}\right)$, ranging from -1 to 1 , and shows the deviation of the multifractal spectrum to the left or to the right. All the above parameters extracted from multifractal analysis of 38 dolostone samples are shown in Table 2.

The multifractal analysis results of samples with different pore structure are shown in Figure 5, respectively. For all the samples, the spectrum $\alpha-q$ of pore distribution is an anti- "S" shape. With increasing $q$, the singularity exponent $\alpha$ is reduced with $q$ and tends to be constant at the minimum and maximum values of $q$ (Figure 5(a)). The mass exponent spectrum $\tau(q)-q$, which increases with the addition of $q$ but not at a constant gradient, shows that the pore space 
TABLE 2: Petrophysical properties and parameters of multifractal analysis on dolostone samples.

\begin{tabular}{|c|c|c|c|c|c|c|c|c|c|c|}
\hline Sample & $\alpha_{\mathrm{L}}$ & $\alpha_{\mathrm{R}}$ & $\Delta \alpha$ & $\Delta \alpha_{\mathrm{L}}$ & $\Delta \alpha_{\mathrm{R}}$ & AI & $\Delta f$ & $D_{0}$ & $D_{1}$ & $D_{2}$ \\
\hline S1 & 1.246 & 1.706 & 0.460 & 1.246 & 0.251 & -0.109 & 0.101 & 1.360 & 1.298 & 1.270 \\
\hline S2 & 1.358 & 1.862 & 0.504 & 1.358 & 0.294 & -0.193 & 0.104 & 1.483 & 1.429 & 1.400 \\
\hline S3 & 1.455 & 1.861 & 0.406 & 1.455 & 0.321 & -0.565 & 0.336 & 1.528 & 1.476 & 1.460 \\
\hline S4 & 1.425 & 1.943 & 0.518 & 1.425 & 0.334 & -0.288 & 0.177 & 1.524 & 1.475 & 1.453 \\
\hline S5 & 1.527 & 2.020 & 0.493 & 1.527 & 0.352 & -0.455 & 0.315 & 1.584 & 1.544 & 1.532 \\
\hline S6 & 1.379 & 1.902 & 0.523 & 1.379 & 0.264 & -0.011 & 0.066 & 1.539 & 1.466 & 1.425 \\
\hline S7 & 1.215 & 1.710 & 0.495 & 1.215 & 0.141 & 0.427 & -0.393 & 1.499 & 1.429 & 1.365 \\
\hline S8 & 1.125 & 1.559 & 0.434 & 1.125 & 0.099 & 0.570 & -0.358 & 1.386 & 1.309 & 1.245 \\
\hline S9 & 1.466 & 1.967 & 0.501 & 1.466 & 0.333 & -0.351 & 0.245 & 1.544 & 1.496 & 1.479 \\
\hline S10 & 1.287 & 1.795 & 0.508 & 1.287 & 0.232 & 0.085 & -0.056 & 1.464 & 1.391 & 1.348 \\
\hline S11 & 1.179 & 1.672 & 0.493 & 1.179 & 0.189 & 0.234 & -0.054 & 1.363 & 1.266 & 1.216 \\
\hline S12 & 1.044 & 1.493 & 0.448 & 1.044 & 0.122 & 0.466 & -0.346 & 1.293 & 1.222 & 1.169 \\
\hline $\mathrm{S} 13$ & 1.511 & 2.032 & 0.520 & 1.511 & 0.349 & -0.346 & 0.185 & 1.610 & 1.569 & 1.548 \\
\hline S14 & 1.489 & 2.039 & 0.549 & 1.489 & 0.335 & -0.213 & 0.102 & 1.623 & 1.569 & 1.538 \\
\hline S15 & 1.201 & 1.626 & 0.425 & 1.201 & 0.178 & 0.163 & -0.038 & 1.359 & 1.293 & 1.253 \\
\hline S16 & 1.307 & 1.806 & 0.499 & 1.307 & 0.221 & 0.111 & -0.082 & 1.493 & 1.421 & 1.374 \\
\hline S17 & 1.130 & 1.543 & 0.414 & 1.130 & 0.096 & 0.537 & -0.560 & 1.407 & 1.365 & 1.324 \\
\hline S18 & 1.159 & 1.642 & 0.483 & 1.159 & 0.124 & 0.487 & -0.459 & 1.464 & 1.401 & 1.336 \\
\hline S19 & 1.272 & 1.766 & 0.495 & 1.272 & 0.199 & 0.190 & -0.080 & 1.466 & 1.382 & 1.329 \\
\hline S20 & 1.347 & 1.778 & 0.431 & 1.347 & 0.204 & 0.012 & -0.016 & 1.508 & 1.450 & 1.410 \\
\hline S21 & 1.239 & 1.727 & 0.488 & 1.239 & 0.186 & 0.230 & -0.115 & 1.443 & 1.359 & 1.306 \\
\hline S22 & 1.300 & 1.686 & 0.386 & 1.300 & 0.149 & 0.242 & -0.113 & 1.466 & 1.408 & 1.369 \\
\hline S23 & 1.127 & 1.654 & 0.527 & 1.127 & 0.303 & -0.150 & 0.192 & 1.302 & 1.251 & 1.204 \\
\hline S24 & 1.475 & 1.912 & 0.437 & 1.475 & 0.262 & -0.249 & 0.139 & 1.557 & 1.525 & 1.497 \\
\hline S25 & 1.293 & 1.674 & 0.382 & 1.293 & 0.180 & 0.067 & 0.041 & 1.424 & 1.364 & 1.330 \\
\hline S26 & 1.271 & 1.722 & 0.451 & 1.271 & 0.188 & 0.162 & -0.053 & 1.441 & 1.367 & 1.323 \\
\hline S27 & 1.174 & 1.647 & 0.474 & 1.174 & 0.165 & 0.296 & -0.202 & 1.401 & 1.325 & 1.267 \\
\hline S28 & 1.369 & 1.833 & 0.464 & 1.369 & 0.224 & 0.047 & -0.004 & 1.528 & 1.468 & 1.429 \\
\hline S29 & 1.073 & 1.531 & 0.458 & 1.073 & 0.135 & 0.413 & -0.254 & 1.312 & 1.232 & 1.171 \\
\hline S30 & 1.185 & 1.583 & 0.398 & 1.185 & 0.258 & -0.346 & 0.204 & 1.277 & 1.241 & 1.221 \\
\hline S31 & 1.267 & 1.654 & 0.387 & 1.267 & 0.172 & 0.068 & -0.028 & 1.401 & 1.340 & 1.307 \\
\hline S32 & 1.457 & 1.979 & 0.522 & 1.457 & 0.216 & 0.171 & -0.221 & 1.560 & 1.542 & 1.513 \\
\hline S33 & 1.469 & 2.017 & 0.548 & 1.469 & 0.259 & 0.056 & -0.126 & 1.597 & 1.622 & 1.576 \\
\hline S34 & 1.235 & 1.605 & 0.371 & 1.235 & 0.110 & 0.460 & -0.301 & 1.439 & 1.388 & 1.347 \\
\hline S35 & 1.167 & 1.588 & 0.421 & 1.167 & 0.102 & 0.510 & -0.318 & 1.410 & 1.338 & 1.282 \\
\hline S36 & 1.250 & 1.631 & 0.381 & 1.250 & 0.090 & 0.527 & -0.073 & 1.463 & 1.366 & 1.315 \\
\hline S37 & 1.496 & 1.846 & 0.350 & 1.496 & 0.165 & -0.028 & 0.012 & 1.586 & 1.556 & 1.520 \\
\hline S38 & 1.447 & 1.840 & 0.393 & 1.447 & 0.170 & 0.115 & -0.013 & 1.680 & 1.520 & 1.478 \\
\hline
\end{tabular}

distributions have multifractal properties (Figure 5(b)). On the multifractal spectrum $f(\alpha)-\alpha, f$ increases with increasing $\alpha$ when $\alpha$ is less than $\alpha_{0}$ but decreases when $\alpha$ is greater than $\alpha_{0}$. The shape of spectrum $f(\alpha)-\alpha$ displays a "left hook," "right hook," or a symmetric parabola (Figure 5(c)).

3.4. Reservoir Heterogeneity Evaluation of Dolomite with Multifractal Analysis. Multifractal theory has been widely used in sandstone reservoir evaluation, pore structure analysis, porosity prediction, and heterogeneity studies $[11,17-$ 19]. Based on multifractal analysis, this work includes esti- mation of porosity for deep-buried dolomite, evaluation of heterogeneity for different types of dolomite, and classification of pore structure for dolostone samples using clustering analysis method and multifractal parameters.

3.4.1. Porosity Estimated with Multifractal Analysis. The crossplot in Figure 6 shows the relationship between the porosity calculated with the capacity dimension and porosity values obtained from core plug helium porosimetry. The results indicated that the porosity values predicted by the capacity dimension are very close to the measured values. 


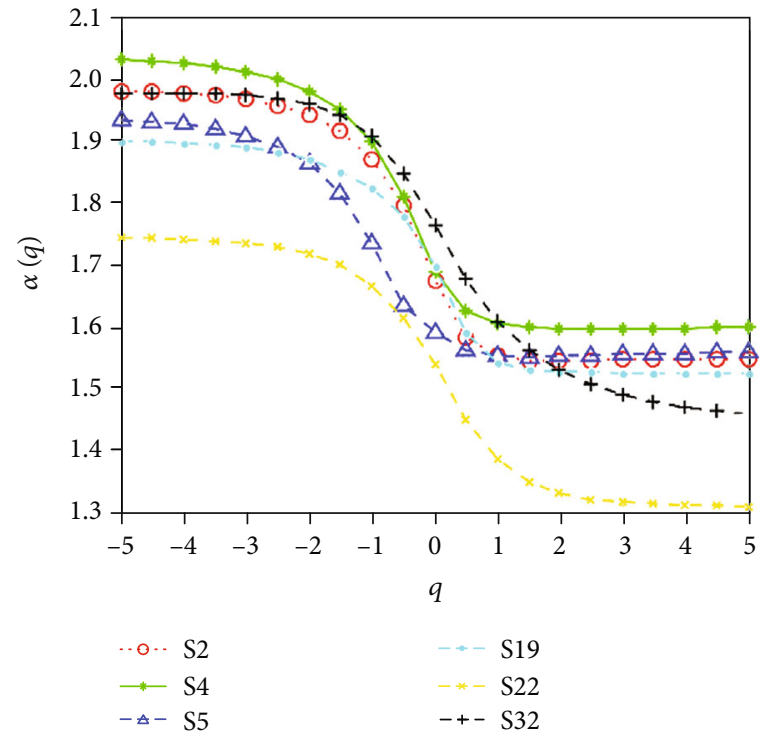

(a)

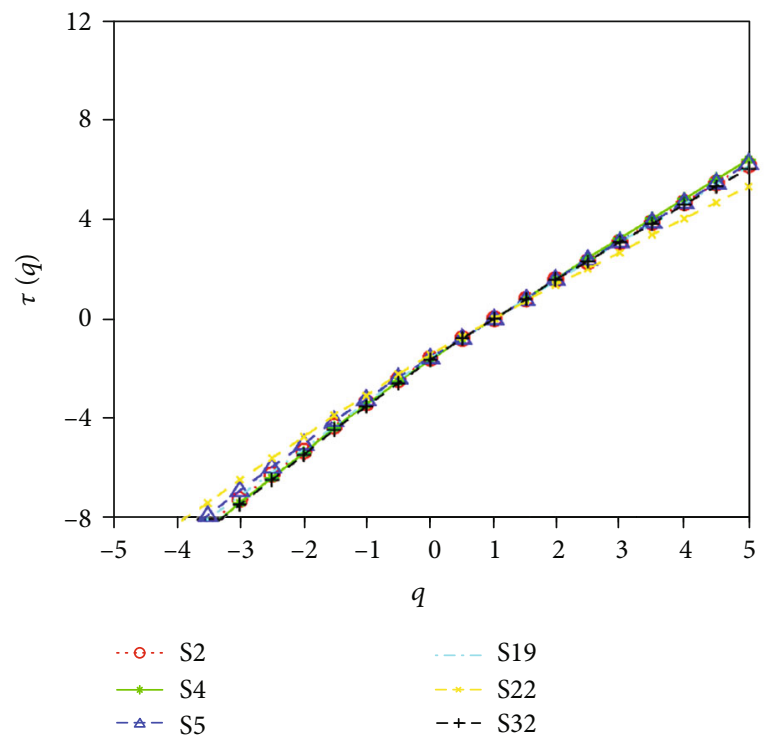

(c)

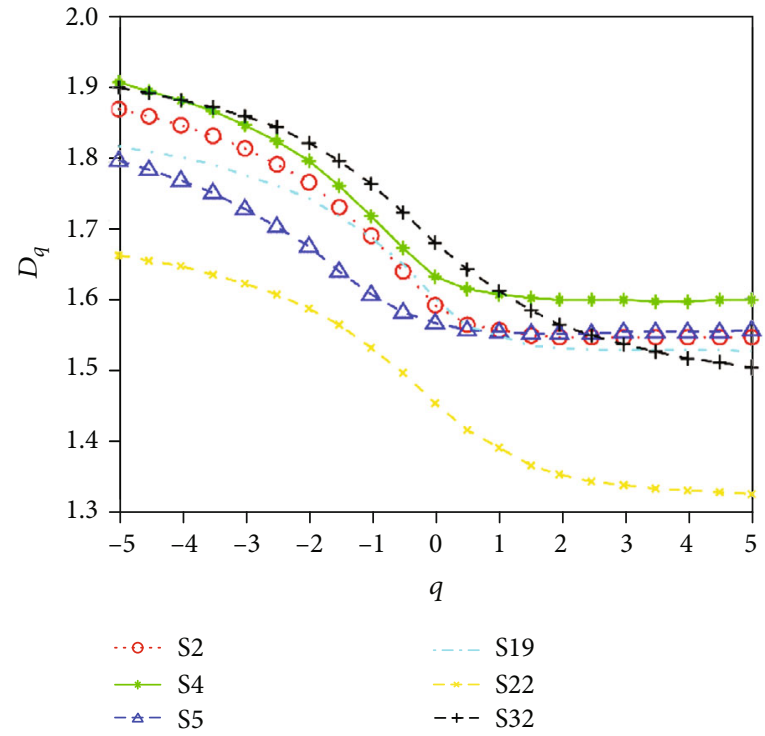

(b)

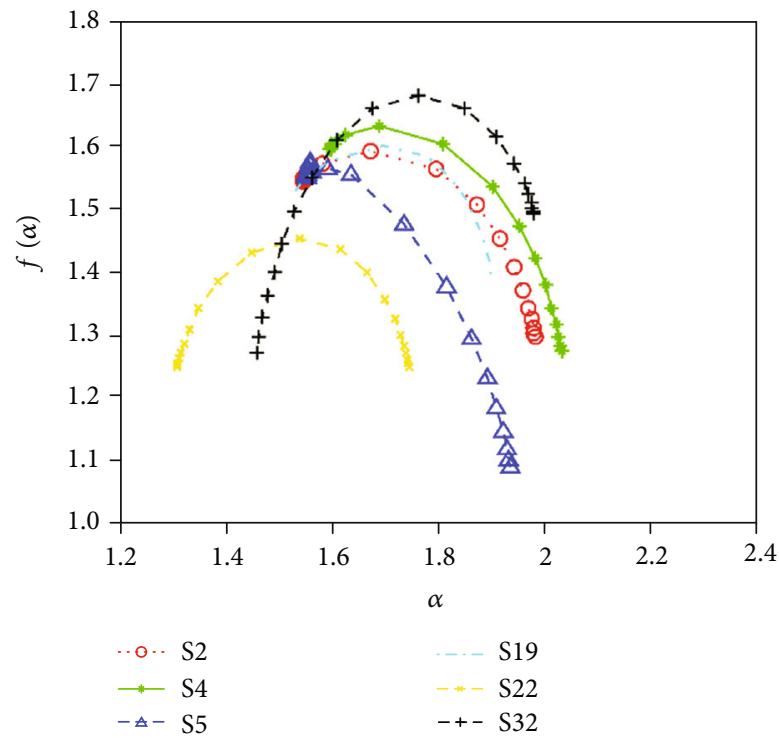

(d)

FIgure 5: Multifractal analysis of pores in dolomite for samples S2, S4, S5, S19, S22, and S32. (a) Plots of singularity exponents against moments $q$. For each sample, the $\alpha(q)$ decreases against the increase of $q$. (b) Plots of $D_{q}$ changing with $q$. (c) Plots of mass exponent varying with the moment $q$. (d) Multifractal spectrum of the Hausdorff dimension $f(a)$ changing with singularity exponent $\alpha$.

Although there is a deviation between the predicted and measured values in two points that may be caused by the selected domain for image from thin section or the heterogeneity of pore space, the predicted porosity is still strongly correlated with measured values with multiple correlation coefficient value 0.865 . Thus, the generalized fractal dimension obtained by multifractal analysis can provide a new method for the porosity prediction of dolomite.

3.4.2. Multifractal Characteristics of Different Types of Dolomite. In order to compare the multifractal characteristics and physical properties of pore spaces in dolostone samples with different crystal sizes, the average values of the multi- fractal and physical parameters extracted from image analysis of each sample were calculated from the data listed in Table 2, and the heterogeneity and physical characteristics of dolomites with different crystal sizes were analyzed. Statistical analyses of these parameters clearly show the differences between various dolomite types. As shown in Table 3, the fine and medium crystalline dolomites have higher values of porosity, permeability, average pore radius, and average pore throat radius than coarse crystalline, silty crystalline, and microcrystalline dolomites. In addition, the multifractal parameters obtained from the fine and medium crystalline dolostone samples are significantly different to those from dolomites with other crystal sizes; e.g., the characteristics of 


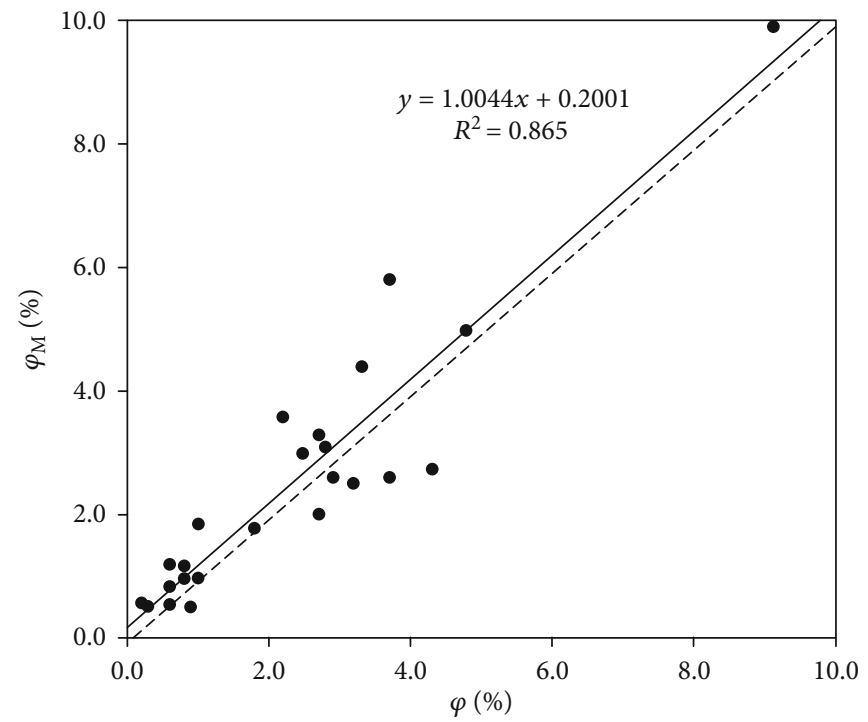

Figure 6: Comparison of total porosity values obtained from core plug helium porosimetry (horizontal axis) and porosity obtained from multifractal analysis on digital image.

TABle 3: Average value of physical properties and multifractal parameters corresponding to different types of dolomites.

\begin{tabular}{lcccccccccccc}
\hline Lithology & $\varphi_{\mathrm{I}}(\%)$ & $R_{\mathrm{b}}(\mu \mathrm{m})$ & $R_{\mathrm{t}}(\mu \mathrm{m})$ & $K_{\mathrm{I}}(\mathrm{mD})$ & $\alpha_{\mathrm{L}}$ & $\alpha_{\mathrm{R}}$ & $\Delta \alpha$ & $\Delta \alpha_{\mathrm{L}}$ & $\Delta \alpha_{\mathrm{R}}$ & $\mathrm{AI}$ & $\Delta f$ & $D_{0}$ \\
\hline $\mathrm{DM}$ & 1.08 & 54.21 & 23.34 & 0.31 & 1.15 & 1.59 & 0.44 & 0.33 & 0.11 & 0.51 & -0.43 & 1.42 \\
$\mathrm{SD}$ & 4.62 & 79.15 & 20.90 & 0.50 & 1.27 & 1.74 & 0.47 & 0.27 & 0.20 & 0.16 & -0.10 & 1.46 \\
$\mathrm{FD}$ & 7.93 & 138.95 & 32.56 & 1.97 & 1.37 & 1.85 & 0.47 & 0.23 & 0.25 & -0.05 & 0.05 & 1.52 \\
$\mathrm{MD}$ & 7.53 & 120.00 & 30.50 & 1.00 & 1.37 & 1.84 & 0.47 & 0.19 & 0.28 & -0.21 & 0.14 & 1.49 \\
$\mathrm{CD}$ & 2.53 & 44.65 & 11.40 & 0.23 & 1.30 & 1.71 & 0.42 & 0.25 & 0.17 & 0.19 & -0.09 & 1.47 \\
$\mathrm{GD}$ & 1.99 & 70.15 & 19.16 & 0.16 & 1.17 & 1.61 & 0.437 & 0.30 & 0.16 & 0.33 & -0.27 & 1.40 \\
\hline
\end{tabular}

higher $\Delta \alpha$ and $D_{0}$ values, negative AI value, and positive $\Delta f$ value demonstrate that they show stronger heterogeneity and comparatively well-developed macropores.

\section{Discussion}

4.1. Magnification Effect on Multifractal Analysis. In addition, the fractal law will be affected by image magnification due to the image zoom setting, and the multifractal parameters will vary with image magnification $[26,43,44,54]$. It can be observed that the fractal dimension and multifractal spectrum change irregularly when the image resolution is changed [26]. In other words, a rock may show complete multifractal or local multifractal characteristics. Multifractality may be found in pore space or in solid matrix. These complex properties may lead to difficulty in identifying the fractal structure from rock images $[43,44]$.

Before conducting multifractal analysis on images, the size of these images should be kept constant by image resampling because the number of pixels influences the results of multifractal analysis. Due to dependence of the minimum distinguished pore scale on the image resolution, the accurate determination of porosity will be directly affected by the pixels of the image if the field of view has been confirmed. In other words, some pores cannot be distinguished at low resolutions, such that the porosity will be lower because the indistinguishable pores are not calculated. In addition, the multifractal spectrum will be different when the number of pixels in the image is changed. In fact, the magnification effect has a direct influence on multifractal analysis [43, 44]. Therefore, in order to compare the multifractal characteristics of pores in different reservoirs, the image scale and number of pixels should be maintained at the same magnification and size.

4.2. Relationship between Petrophysical Properties and Multifractal Parameters. The pore structure can be described by a continuous distribution series of singularity exponents $\alpha$ . Therefore, the values $\alpha_{\min }$ and $\alpha_{\max }$ correspond to the maximum and minimum pore sizes, respectively. In Figure 7(a), there is a strong positive correlation between porosity and the value of $\alpha_{\min }$ and $\alpha_{\max }$, where the value of $\alpha_{\min }$ and $\alpha_{\max }$ increases gradually with increasing porosity. Figure $8(a)$ shows a slightly nonlinear correlation between permeability and $\alpha_{\min }$ and $\alpha_{\max }$.

The value of $\Delta \alpha$, as a comprehensive parameter for evaluating the heterogeneity of the spatial distribution of pores, reflects the degree of aggregation in pore scale. The higher the value of $\Delta \alpha$, the more dispersive the pore size distribution, which indicates strong heterogeneity of the pore space 


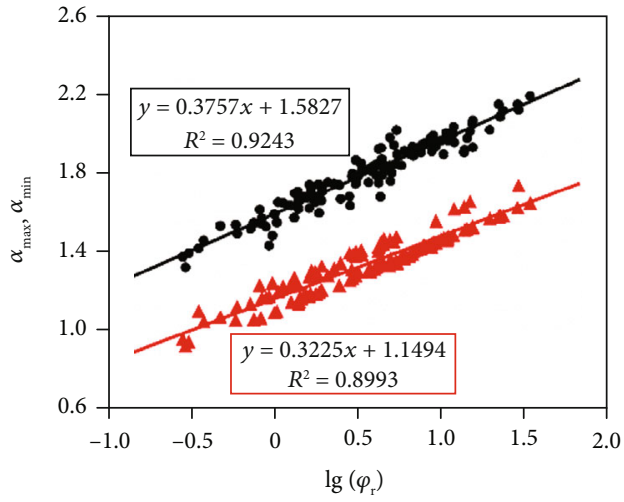

- $\alpha_{\max }$

$\Delta \alpha_{\min }$

(a)

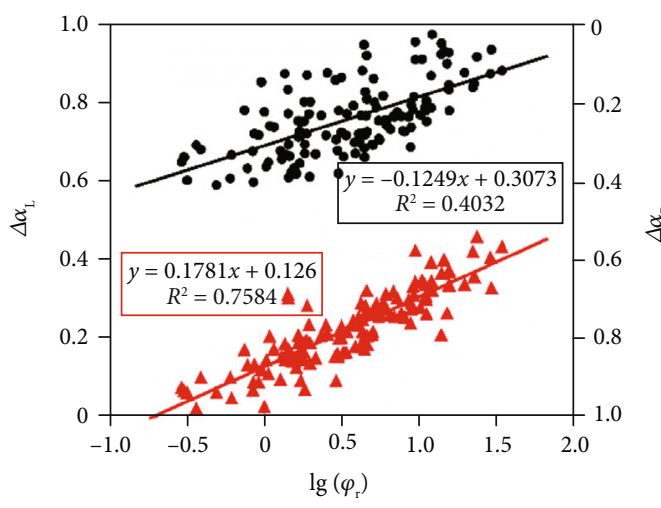

$\Delta \Delta \alpha_{\mathrm{R}}$

(c)

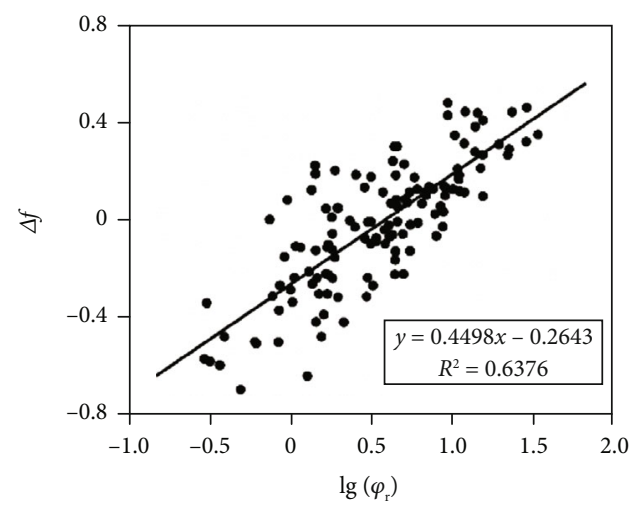

(e)

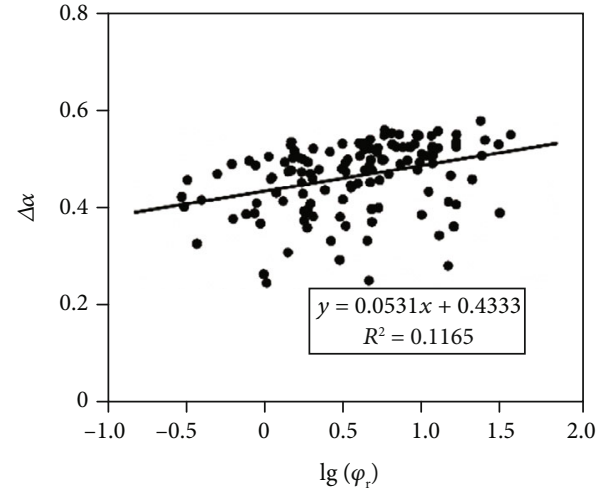

(b)

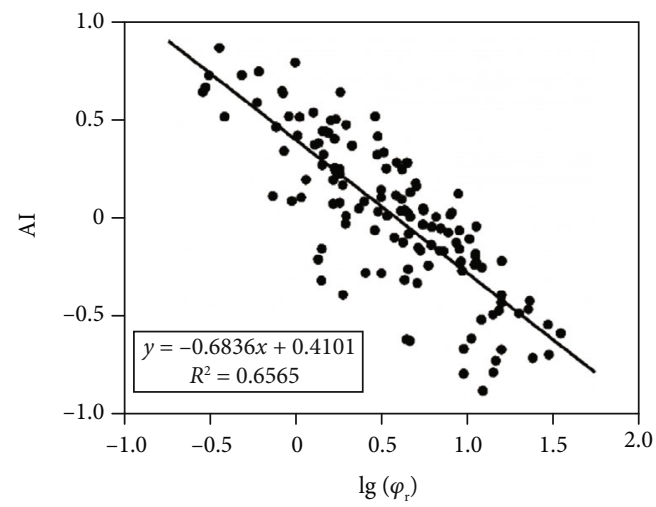

(d)

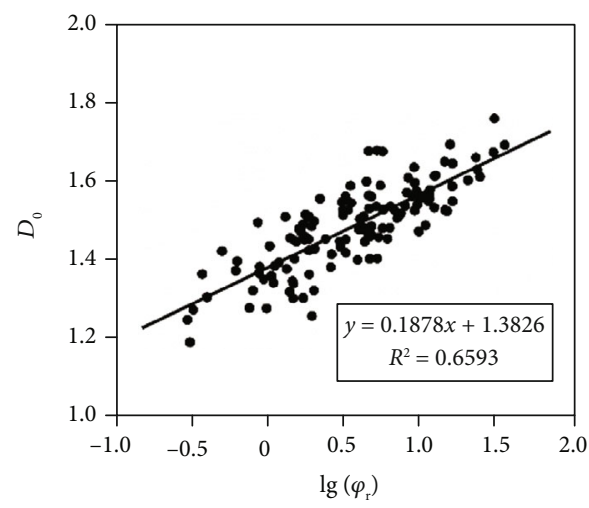

(f)

Figure 7: The crossplots of porosity values and multifractal parameters. (a) The relationship between singularity exponents $\alpha_{\min }$ and $\alpha_{\max }$ and porosity from image analysis. (b) The relationship between $\Delta \alpha$ and porosity from image analysis. (c) The relationship between $\Delta \alpha_{\mathrm{L}}, \Delta \alpha_{\mathrm{R}}$, and porosity from image analysis. (d) The relationship between AI and porosity. (e) The relationship between $\Delta f$ and porosity. (f) The relationship between $D_{0}$ and porosity.

in dolomite. In addition, as there are no significant correlations between the porosity, permeability, and value of $\Delta \alpha$, it indicates that the influence of reservoir heterogeneity on petrophysical properties is complex and cannot be described with a simple linear formula (Figures $7(\mathrm{~b})$ and $8(\mathrm{~b}))$. However, there is a positive correlation between $\Delta$ $\alpha_{\mathrm{L}}$ and porosity but a strong negative correlation between
$\Delta \alpha_{\mathrm{R}}$ and porosity, which indicates higher porosity and pores concentrated in a larger pore size range (Figures 7(c) and 8(c)). Nevertheless, there is only a slightly linear relationship between $\Delta \alpha_{\mathrm{L}}, \Delta \alpha_{\mathrm{R}}$, and permeability.

The asymmetrical index (AI), as a parameter to describe the regularity of the multifractal spectrum, is determined by the difference between the values of $\Delta \alpha_{\mathrm{L}}$ and $\Delta \alpha_{\mathrm{R}}$. A positive 


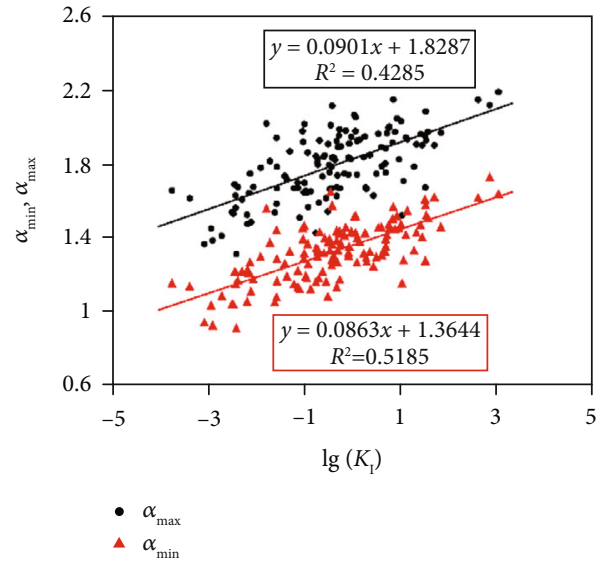

(a)

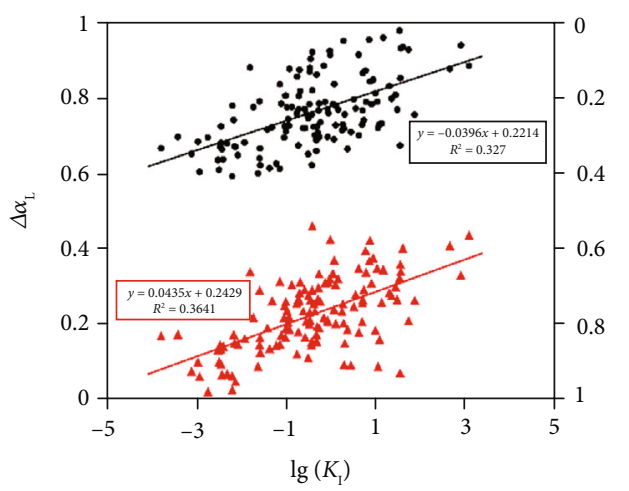

$\Delta \Delta \alpha_{\mathrm{R}}$

- $\Delta \alpha_{\mathrm{L}}$

(c)

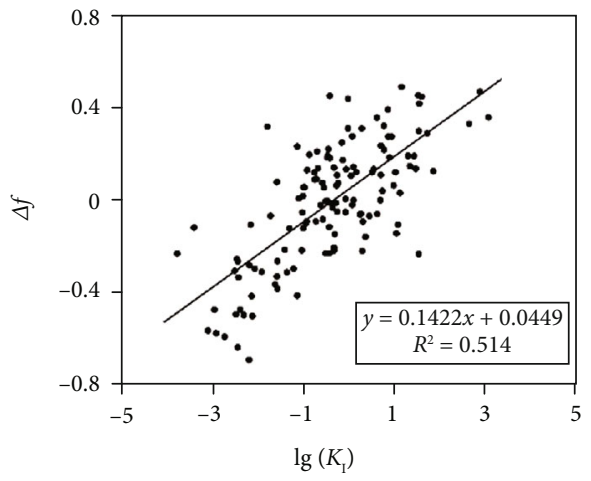

(e)

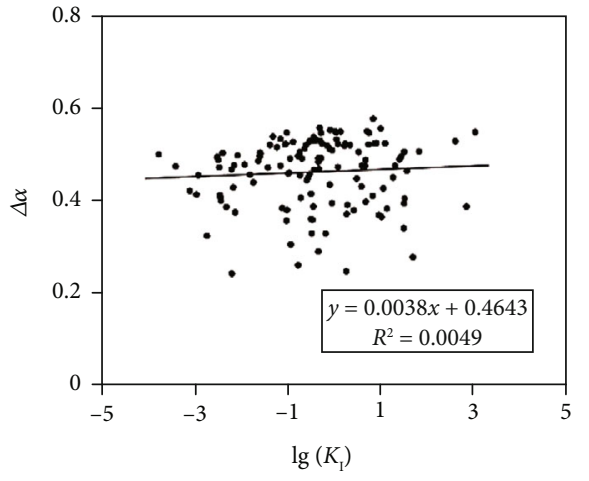

(b)

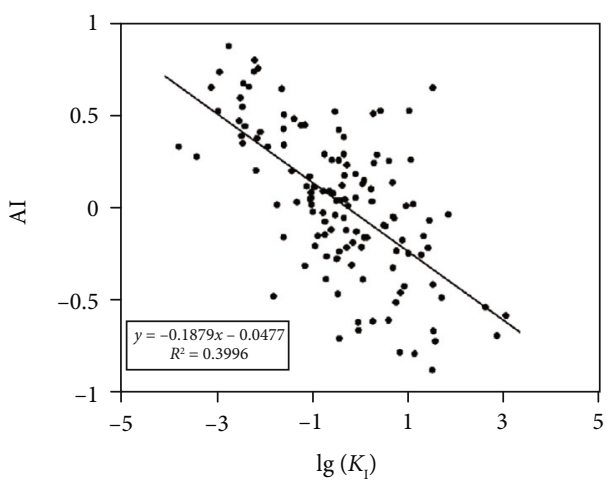

(d)

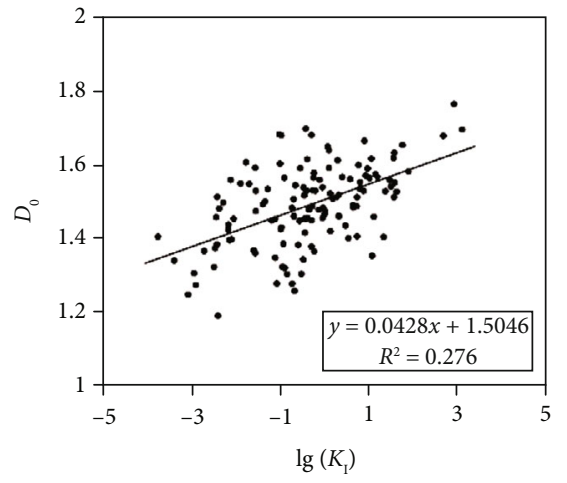

(f)

FiguRE 8: The crossplots of permeability values and multifractal parameters. (a) The relationship between singularity exponents $\alpha_{\min }$ and $\alpha_{\text {max }}$ and permeability from image analysis. (b) The relationship between $\Delta \alpha$ and permeability from image analysis. (c) The relationship between $\Delta \alpha_{\mathrm{L}}, \Delta \alpha_{\mathrm{R}}$, and permeability from image analysis. (d) The relationship between AI and permeability. (e) The relationship between $\Delta f$ and permeability. (f) The relationship between $D_{0}$ and permeability.

value of AI indicates that the pores are scattered dispersively in the large-scale range but concentrated in the small-scale range, and vice versa. Generally, the corresponding value of porosity is larger when the pore size distribution is concentrated in the large-scale range. Therefore, a significant negative correlation is presented in the AI-porosity crossplot but there is a slightly negative correlation between $\mathrm{AI}$ and $K_{\mathrm{I}}$ in the AI-permeability crossplot (Figures 7(d) and 8(d)).
The $\Delta f$ parameter reflects the morphological characteristics of the multifractal spectrum. When the value of $\Delta f$ is positive, the $f(\alpha)-\alpha$ curve will display as a "left hook," and vice versa, it will display as a "right hook." As shown in Figures $7(\mathrm{e})$ and $8(\mathrm{e})$, the higher the value of $\Delta f$, the higher the porosity will be. This is mainly because macropores will engender a larger contribution and influence on the value of porosity if they play a dominant role in the reservoir space 
TABLE 4: The statistical results of porosity and permeability in different classes based on $K$-means clustering analysis with multifractal parameters.

\begin{tabular}{|c|c|c|c|c|c|c|}
\hline \multirow{2}{*}{ Type } & \multicolumn{3}{|c|}{ Porosity (\%) } & \multicolumn{3}{|c|}{ Permeability $(\mathrm{mD})$} \\
\hline & Maximum & Minimum & Average & Maximum & Minimum & Average \\
\hline $\mathrm{I}$ & 3.6 & 1.4 & 2.4 & 0.809 & 0.034 & 0.395 \\
\hline II & 8.1 & 2.2 & 5.2 & 3.772 & 0.089 & 1.013 \\
\hline III & 18.4 & 7.5 & 12.1 & 7.365 & 0.558 & 2.658 \\
\hline IV & 2.9 & 0.8 & 1.4 & 2.677 & 0.001 & 0.373 \\
\hline
\end{tabular}

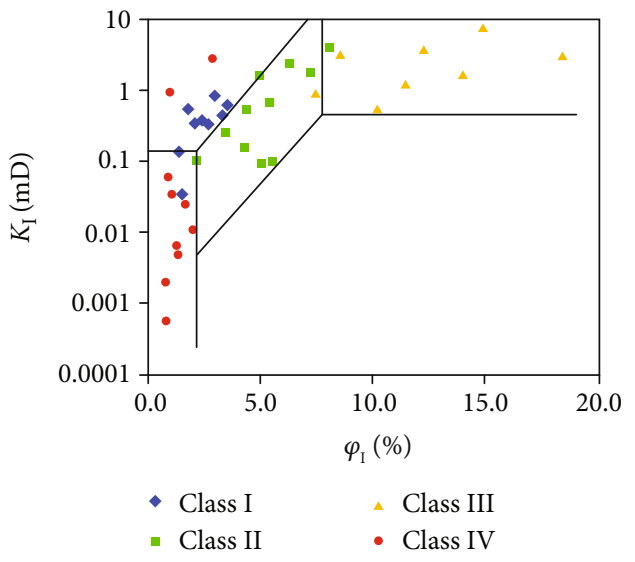

(a)

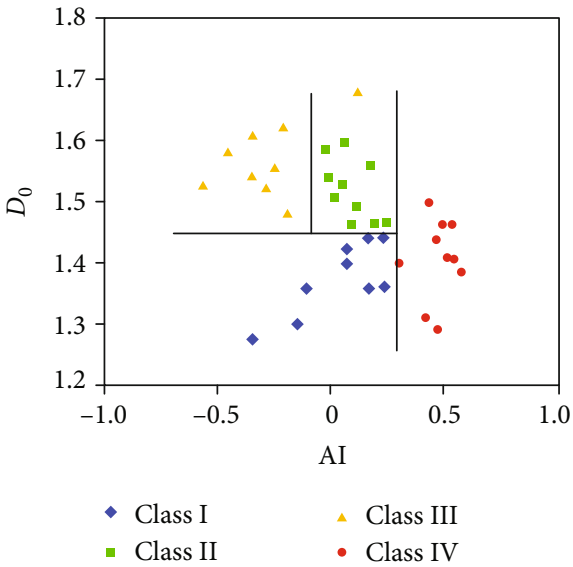

(b)

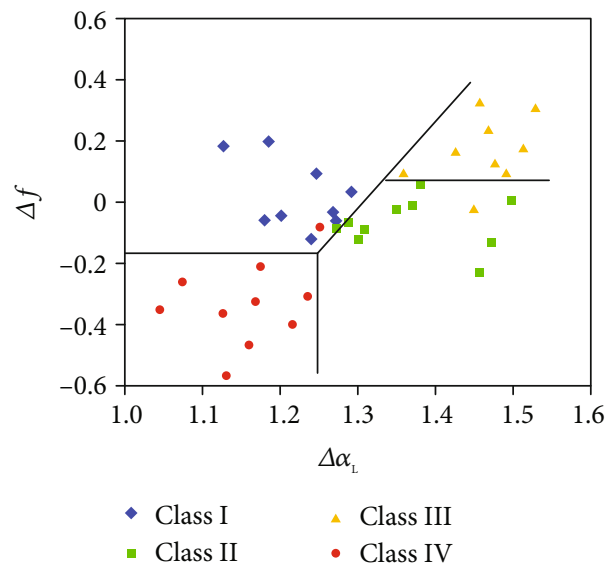

(c)

FIgURE 9: Crossplot of porosity and permeability and multifractal feature parameters of dolomites in different categories from $K$-means clustering analysis based on multifractal parameters.

of a rock. In addition, there is a correlation preference between the value of $D_{0}$ and porosity, but not with permeability (Figures 7(f) and 8(f)).

\subsection{Reservoir Evaluation Based on K-Means Clustering with} Multifractal Parameters. In this study, $K$-means clustering analysis was introduced to fully exploit the reservoir implications of multifractal parameters obtained from the multifractal analysis of digital images.

According to the multifractal parameters of each sample, the $K$-means clustering method was used to classify these samples into four categories. The statistics of physical prop- erties for each category show significant differences between them. As shown in Table 4 and Figure 9, class III corresponds to the best physical reservoir properties, the porosity of which is greater than $7.5 \%$ with an average value of $12.1 \%$ and the permeability of which is generally greater than $0.56 \mathrm{mD}$ with an average value of $2.66 \mathrm{mD}$. For class II, the porosity ranges from $2.2 \%$ to $8.1 \%$ with an average value of $5.2 \%$, while the permeability ranges from $0.089 \mathrm{mD}$ to $3.772 \mathrm{mD}$ with an average value of $1.01 \mathrm{mD}$. Classes I and IV, characterized by low porosity and low permeability pore structure types, have average porosity values of $2.4 \%$ and $1.4 \%$, respectively. Based on the multifractal characteristic parameters, the $K$-means 
clustering analysis method can be used to classify reservoirs and provide a new method for reservoir evaluation and classification of pore structure.

Based on the parameters such as $D_{0}, \mathrm{AI}, \Delta \alpha_{\mathrm{L}}$, and $\Delta f$, we attempted to construct the criteria of pore structure, with purpose of pore structure classification by multifractal analysis. Figure 9 shows the crossplot of porosity and permeability, $D_{0}$ and $\mathrm{AI}$, and $\Delta \alpha_{\mathrm{L}}$ and $\Delta f$. We establish the discrimination criteria for pore typing, which are expressed as follows:

Pore structure class $I: \mathrm{AI}<0.24$ and $D_{0} \leq 1.44$ and $\Delta f>$ -0.11 .

Pore structure class II: $-0.03<\mathrm{AI}<0.24, D_{0}>1.44, \Delta f$ $<0.07$, and $\Delta \alpha_{\mathrm{L}}>1.27$.

Pore structure class III: $\mathrm{AI} \leq-0.03, D_{0}>1.44, \Delta f \geq 0.07$, and $\Delta \alpha_{\mathrm{L}} \geq 1.36$.

Pore structure class $I V: \mathrm{AI} \geq 0.24, \Delta f<-0.11$, and $\Delta \alpha_{\mathrm{L}}$ $\leq 1.27$.

\section{Conclusions}

In this study, multifractal analysis was used to evaluate the pore space in dolomite reservoirs. Then, the relationship between the statistical multifractal characteristic parameters and the physical properties of dolomite was analyzed. Finally, the pore structure of dolomite was classified by $K$-means clustering analysis with multifractal parameters. The conclusions can be summarized as follows:

(1) The porosity and permeability of deep-buried dolomite are closely related to the multifractal characteristic parameters. There are strong correlations between porosity, permeability, and multifractal parameters, such as $\alpha_{\text {min }}, \alpha_{\text {max }}, \Delta \alpha_{\mathrm{R}}, \Delta \alpha_{\mathrm{L}}$, AI, and $\Delta$ $f$. The porosity can be accurately predicted on the basis of the multifractal generalized dimension, and this provides a new method for porosity estimation of dolomite reservoirs from thin section images

(2) Significant divergence in porosity, permeability, and multifractal parameters has been found between dolomites with different crystal sizes, since the rock type and crystallization degree control the reservoir performance and permeability of dolomite to a certain extent

(3) $K$-means clustering analysis of multifractal feature parameters can be used for quantitative pore structure classification and precise reservoir evaluation. The discrimination criteria for pore typing can be expressed with AI, $D_{0}, \Delta f$, and $\Delta \alpha_{\mathrm{L}}$

\section{Data Availability}

The data used to support the findings of this study are included within the article.

\section{Conflicts of Interest}

The authors declare that they have no conflicts of interest.

\section{Acknowledgments}

Funding for this research was provided by Key Laboratory of Reservoir Characterization, CNPC. The samples and thin sections are obtained with help from Exploration and Development Research Institute of Sinopec Northwest Oilfield Company. The image collection and data analysis were completed in State Key Laboratory of Oil and Gas Reservoir Geology and Exploitation, Southwest Petroleum University, China. We also thank Professor Cai Jianchao, China University of Geosciences (Wuhan), for his examining of the manuscript and precious revision suggestions.

\section{References}

[1] J. Lai, S. Wang, C. Zhang et al., "Spectrum of pore types and networks in the deep Cambrian to Lower Ordovician dolostones in Tarim Basin, China," Marine and Petroleum Geology, vol. 112, article 104081, 2020.

[2] J. Lai, S. Wang, G. Wang et al., "Pore structure and fractal characteristics of Ordovician Majiagou carbonate reservoirs in Ordos Basin, China," AAPG Bulletin, vol. 103, no. 11, pp. 2573-2596, 2019.

[3] J. H. Norbisrath, G. P. Eberli, B. Laurich, G. Desbois, R. J. Weger, and J. L. Urai, "Electrical and fluid flow properties of carbonate microporosity types from multiscale digital image analysis and mercury injection," AAPG Bulletin, vol. 99, no. 11, pp. 2077-2098, 2015.

[4] J. Lai, G. Wang, Z. Fan et al., "Insight into the pore structure of tight sandstones using NMR and HPMI measurements," Energy \& Fuels, vol. 30, no. 12, pp. 10200-10214, 2016.

[5] B. B. Mandelbrot and J. A. Wheeler, "The fractal geometry of nature," American Journal of Physics, vol. 51, no. 3, pp. 286287, 1983.

[6] A. J. Katz and A. H. Thompson, "Fractal sandstone pores: implications for conductivity and pore formation," Physical Review Letters, vol. 54, no. 12, pp. 1325-1328, 1985.

[7] H. Pape, L. Riepe, and J. R. Schopper, "Theory of self-similar network structures in sedimentary and igneous rocks and their investigation with microscopical and physical methods," Journal of Microscopy, vol. 148, no. 2, pp. 121-147, 1987.

[8] A. H. Thompson, A. J. Katz, and C. E. Krohn, "The microgeometry and transport properties of sedimentary rock," Advances in Physics, vol. 36, no. 5, pp. 625-694, 1987.

[9] J. W. Crawford and N. Matsui, "Heterogeneity of the pore and solid volume of soil: distinguishing a fractal space from its non-fractal complement," Geoderma, vol. 73, no. 3-4, pp. 183-195, 1996.

[10] J. Lai and G. Wang, "Fractal analysis of tight gas sandstones using high-pressure mercury intrusion techniques," Journal of Natural Gas Science and Engineering, vol. 24, pp. 185-196, 2015.

[11] Z. Song, G. Liu, W. Yang, H. Zou, M. Sun, and X. Wang, "Multi-fractal distribution analysis for pore structure characterization of tight sandstone-a case study of the Upper Paleozoic tight formations in the Longdong District, Ordos Basin," Marine and Petroleum Geology, vol. 92, pp. 842-854, 2018.

[12] X. Chen, G. Yao, J. Cai, Y. Huang, and X. Yuan, "Fractal and multifractal analysis of different hydraulic flow units based on micro-CT images," Journal of Natural Gas Science and Engineering, vol. 48, pp. 145-156, 2017. 
[13] Y. Xia, J. Cai, E. Perfect, W. Wei, Q. Zhang, and Q. Meng, "Fractal dimension, lacunarity and succolarity analyses on CT images of reservoir rocks for permeability prediction," Journal of Hydrology, vol. 579, article 124198, 2019.

[14] Y. Wu, P. Tahmasebi, C. Lin et al., "A comprehensive study on geometric, topological and fractal characterizations of pore systems in low-permeability reservoirs based on SEM, MICP, NMR, and X-ray CT experiments," Marine and Petroleum Geology, vol. 103, pp. 12-28, 2019.

[15] B. B. Mandelbrot, "Multifractal measures, especially for the geophysicist," Pure and Applied Geophysics, vol. 131, no. 1-2, pp. 5-42, 1989.

[16] S. Xie, Q. Cheng, Q. Ling, B. Li, Z. Bao, and P. Fan, "Fractal and multifractal analysis of carbonate pore-scale digital images of petroleum reservoirs," Marine and Petroleum Geology, vol. 27, no. 2, pp. 476-485, 2010.

[17] X. Ge, Y. Fan, J. Li, and M. Aleem Zahid, "Pore structure characterization and classification using multifractal theory-an application in Santanghu basin of western China," Journal of Petroleum Science and Engineering, vol. 127, pp. 297-304, 2015.

[18] P. Zhao, Z. Wang, Z. Sun, J. Cai, and L. Wang, "Investigation on the pore structure and multifractal characteristics of tight oil reservoirs using NMR measurements: Permian Lucaogou Formation in Jimusaer Sag, Junggar Basin," Marine and Petroleum Geology, vol. 86, pp. 1067-1081, 2017.

[19] J. Peng, H. Han, Q. Xia, and B. Li, "Evaluation of the pore structure of tight sandstone reservoirs based on multifractal analysis: a case study from the Kepingtage Formation in the Shuntuoguole Uplift, Tarim Basin, NW China," Journal of Geophysics and Engineering, vol. 15, no. 4, pp. 1122-1136, 2018.

[20] P. Zhao, X. Wang, J. Cai et al., "Multifractal analysis of pore structure of Middle Bakken formation using low temperature $\mathrm{N}_{2}$ adsorption and NMR measurements," Journal of Petroleum Science and Engineering, vol. 176, pp. 312-320, 2019.

[21] J. Piñuela, A. Alvarez, D. Andina, R. J. Heck, and A. M. Tarquis, "Quantifying a soil pore distribution from 3D images: multifractal spectrum through wavelet approach," Geoderma, vol. 155, no. 3-4, pp. 203-210, 2010.

[22] F. San José Martínez, M. A. Martín, F. J. Caniego et al., "Multifractal analysis of discretized X-ray CT images for the characterization of soil macropore structures," Geoderma, vol. 156, no. 1-2, pp. 32-42, 2010

[23] J. A. Lafond, L. Han, S. E. Allaire, and P. Dutilleul, "Multifractal properties of porosity as calculated from computed tomography (CT) images of a sandy soil, in relation to soil gas diffusion and linked soil physical properties," European Journal of Soil Science, vol. 63, no. 6, pp. 861-873, 2012.

[24] Y. Gao, J. Jiang, G. de Schutter, G. Ye, and W. Sun, "Fractal and multifractal analysis on pore structure in cement paste," Construction and Building Materials, vol. 69, no. 11, pp. 253-261, 2014.

[25] A. Dathe and P. Baveye, "Dependence of the surface fractal dimension of soil pores on image resolution and magnification," European Journal of Soil Science, vol. 54, no. 3, pp. 453-466, 2003.

[26] A. Dathe, A. M. Tarquis, and E. Perrier, "Multifractal analysis of the pore- and solid-phases in binary two-dimensional images of natural porous structures," Geoderma, vol. 134, no. 3-4, pp. 318-326, 2006.
[27] E. Vidal-Vázquez, O. A. Camargo, S. R. Vieira et al., "Multifractal analysis of soil properties along two perpendicular transects," Vadose Zone Journal, vol. 12, no. 3, pp. 1-13, 2013.

[28] C. E. Krohn, "Fractal measurements of sandstones, shales, and carbonates," Journal of Geophysical Research, vol. 93, no. B4, pp. 3297-3305, 1988.

[29] J. Muller, O. K. Huseby, and A. Saucier, "Influence of multifractal scaling of pore geometry on permeabilities of sedimentary rocks," Chaos Solitons \& Fractals, vol. 5, no. 8, pp. 14851492, 1995.

[30] S. Karimpouli and P. Tahmasebi, "3D multi-fractal analysis of porous media using 3D digital images: considerations for heterogeneity evaluation," Geophysical Prospecting, vol. 67, pp. 1082-1093, 2018.

[31] L. Zhou and Z. Kang, "Fractal characterization of pores in shales using NMR: a case study from the Lower Cambrian Niutitang Formation in the Middle Yangtze Platform, Southwest China," Journal of Natural Gas Science and Engineering, vol. 35, pp. 860-872, 2016.

[32] P. Zhao, X. Hu, X. Ge, Z. Wang, and J. Cai, Multifractal Analysis of Pore Structure of Tight Oil Reservoirs Using LowField NMR Measurements, Elsevier: Petrophysical Characterization and Fluids Transport in Unconventional Reservoirs, 2019.

[33] L. Luo and H. Lin, "Lacunarity and fractal analyses of soil macropores and preferential transport using micro-X-ray computed tomography," Vadose Zone Journal, vol. 8, no. 1, pp. 233-241, 2009.

[34] J. He, W. Ding, A. Li et al., "Quantitative microporosity evaluation using mercury injection and digital image analysis in tight carbonate rocks: a case study from the Ordovician in the Tazhong Palaeouplift, Tarim Basin, NW China," Journal of Natural Gas Science and Engineering, vol. 34, pp. 627-644, 2016.

[35] P. Li, M. Zheng, H. Bi, S. Wu, and X. Wang, "Pore throat structure and fractal characteristics of tight oil sandstone: a case study in the Ordos Basin, China," Journal of Petroleum Science and Engineering, vol. 149, pp. 665-674, 2017.

[36] J. Chen, E. Li, and J. Luo, "Characterization of microscopic pore structures of rock salt through mercury injection and nitrogen absorption tests," Geofluids, vol. 2018, Article ID 927361, 7 pages, 2018.

[37] B. Yu and J. Li, "Some fractal characters of porous media," Fractals, vol. 9, no. 3, pp. 365-372, 2011.

[38] G. S. Padhy, C. Lemaire, E. S. Amirtharaj, and M. A. Ioannidis, "Pore size distribution in multiscale porous media as revealed by DDIF-NMR, mercury porosimetry and statistical image analysis," Colloids and Surfaces A-Physicochemical and Engineering Aspects, vol. 300, no. 1-2, pp. 222-234, 2007.

[39] P. Xu and B. Yu, "Developing a new form of permeability and Kozeny-Carman constant for homogeneous porous media by means of fractal geometry," Advances in Water Resources, vol. 31, no. 1, pp. 74-81, 2008.

[40] Z. Zhang and A. Weller, "Fractal dimension of pore-space geometry of an Eocene sandstone formation," Geophysics, vol. 79, no. 6, pp. D377-D387, 2014.

[41] J. Cai, D. Lin, H. Singh, S. Zhou, Q. Meng, and Q. Zhang, "A simple permeability model for shale gas and key insights on relative importance of various transport mechanisms," Fuel, vol. 252, pp. 210-219, 2019. 
[42] J. Muller, "Characterization of the North Sea chalk by multifractal analysis," Journal of Geophysical Research, vol. 99, no. B4, pp. 7275-7280, 1994.

[43] M. S. Jouini, S. Vega, and E. A. Mokhtar, "Multiscale characterization of pore spaces using multifractals analysis of scanning electronic microscopy images of carbonates," Nonlinear Processes in Geophysics, vol. 18, no. 6, pp. 941-953, 2011.

[44] S. Vega and M. S. Jouini, "2D multifractal analysis and porosity scaling estimation in Lower Cretaceous carbonates," Geophysics, vol. 80, no. 6, pp. 575-586, 2015.

[45] J. Du and W. Pan, "Accumulation conditions and play targets of oil and gas in the Cambrian subsalt dolomite, Tarim Basin, NW China," Petroleum Exploration and Development, vol. 43, no. 3, pp. 327-339, 2016.

[46] J.-P. Yan, Q. Liang, Z.-Z. Li, B. Geng, X.-P. Kou, and Y. Hu, “A connected domain identification method and its application in quantitatively pickup information of caves using electric imaging logging," Chinese Journal of Geophysics, vol. 59, no. 12, pp. 4759-4770, 2016.

[47] S. A. Flavio, S. Luthi, and G. P. Eberli, "Quantitative characterization of carbonate pore systems by digital image analysis," AAPG Bulletin, vol. 82, no. 10, pp. 1815-1836, 1998.

[48] Y. Wu, P. Tahmasebi, C. Lin, and C. Dong, "Process-based and dynamic 2D modeling of shale samples: considering the geology and pore-system evolution," International Journal of Coal Geology, vol. 218, article 103368, 2020.

[49] Y. Wu, P. Tahmasebi, C. Lin, L. Ren, and C. Dong, "Multiscale modeling of shale samples based on low- and high-resolution images," Marine and Petroleum Geology, vol. 109, pp. 9-21, 2019.

[50] Y. Wu, P. Tahmasebi, C. Lin, M. J. Munawar, and V. Cnudde, "Effects of micropores on geometric, topological and transport properties of pore systems for low-permeability porous media," Journal of Hydrology, vol. 575, pp. 327-342, 2019.

[51] E. Müller-Huber and J. Schön, "Carbonates with complex pore systems-modeling of permeabilities and application of modified capillary tube models," in SEG Technical Program Expanded Abstracts 2013, pp. 2587-2591, Society of Exploration Geophysicists, 2013.

[52] A. Chhabra and R. V. Jensen, "Direct determination of the $f(\alpha)$ singularity spectrum," Physical Review Letters, vol. 62, no. 12, pp. 1327-1330, 1989.

[53] R. J. Dunham, "Classification of carbonate rocks according to depositional texture," AAPG Bulletin, vol. 1, pp. 108-121, 1962.

[54] Z. Liu, B. Shi, H. I. Inyang, and Y. Cai, "Magnification effects on the interpretation of SEM images of expansive soils," Engineering Geology, vol. 78, no. 1-2, pp. 89-94, 2005. 